\title{
Impaired SorLA maturation and trafficking as a new mechanism for SORL 1 missense variants in Alzheimer disease
}

\author{
Anne Rovelet-Lecrux ${ }^{1} \mathbb{B}$, Sebastien Feuillette ${ }^{1+}(\mathbb{D})$, Laetitia Miguel ${ }^{1+} \mathbb{B}^{\mathbb{D}}$, Catherine Schramm $^{1} \mathbb{B}$, Ségolène Pernet ${ }^{1}$,

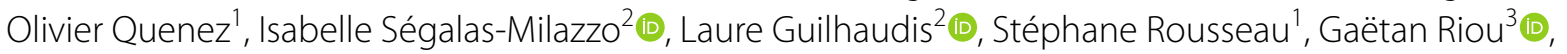

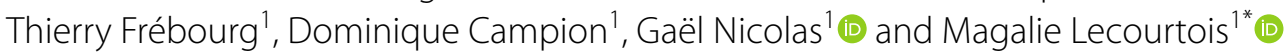

\begin{abstract}
The SorLA protein, encoded by the SORL1 gene, is a major player in Alzheimer's disease (AD) pathophysiology. Functional and genetic studies demonstrated that SorLA deficiency results in increased production of A $\beta$ peptides, and thus a higher risk of AD. A large number of SORL 1 missense variants have been identified in AD patients, but their functional consequences remain largely undefined. Here, we identified a new pathophysiological mechanism, by which rare SORL 1 missense variants identified in AD patients result in altered maturation and trafficking of the SorLA protein. An initial screening, based on the overexpression of 70 SorLA variants in HEK293 cells, revealed that 15 of them (S114R, R332W, G543E, S564G, S577P, R654W, R729W, D806N, Y934C, D1535N, D1545E, P1654L, Y1816C, W1862C, P1914S) induced a maturation and trafficking-deficient phenotype. Three of these variants (R332W, S577P, and R654W) and two maturation-competent variants (S124R and N371T) were further studied in details in CRISPR/ Cas9-modified hiPSCs. When expressed at endogenous levels, the R332W, S577P, and R654W SorLA variants also showed a maturation defective profile. We further demonstrated that these variants were largely retained in the endoplasmic reticulum, resulting in a reduction in the delivery of SorLA mature protein to the plasma membrane and to the endosomal system. Importantly, expression of the R332W and R654W variants in hiPSCs was associated with a clear increase of $A \beta$ secretion, demonstrating a loss-of-function effect of these SorLA variants regarding this ultimate readout, and a direct link with AD pathophysiology. Furthermore, structural analysis of the impact of missense variants on SorLA protein suggested that impaired cellular trafficking of SorLA protein could be due to subtle variations of the protein 3D structure resulting from changes in the interatomic interactions.
\end{abstract}

Keywords: SorLA, iPSC, Alzheimer's disease, Maturation defects, Trafficking

\section{Introduction}

Alzheimer's disease (AD) is a genetically heterogenous disorder. Besides rare families with autosomal dominant early-onset AD (EOAD, onset before 66 years) due to

\footnotetext{
*Correspondence: magalie.lecourtois@univ-rouen.fr

†'Sebastien Feuillette and Laetitia Miguel have contributed equally to this work.

${ }^{1}$ Department of Genetics and CNR-MAJ, Normandie Univ, UNIROUEN, Inserm U1245, CHU Rouen, FHU G4 Génomique, 76000 Rouen, France Full list of author information is available at the end of the article
}

pathogenic variants in APP, PSEN1 or PSEN2, the determinism of $\mathrm{AD}$ is complex and includes a large multigenic component. A wide diversity of genetic risk factors has been identified, varying both in terms of frequencies and strength of effect on AD risk [1]. Exome and genome sequencing recently allowed the identification of three genes harboring a higher burden of rare non-synonymous variants in $\mathrm{AD}$ cases as compared to controls: TREM2, SORL1, and ABCA7 [2].

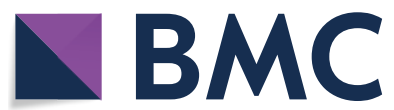

(c) The Author(s) 2021. Open Access This article is licensed under a Creative Commons Attribution 4.0 International License, which permits use, sharing, adaptation, distribution and reproduction in any medium or format, as long as you give appropriate credit to the original author(s) and the source, provide a link to the Creative Commons licence, and indicate if changes were made. The images or other third party material in this article are included in the article's Creative Commons licence, unless indicated otherwise in a credit line to the material. If material is not included in the article's Creative Commons licence and your intended use is not permitted by statutory regulation or exceeds the permitted use, you will need to obtain permission directly from the copyright holder. To view a copy of this licence, visit http://creativecommons.org/licenses/by/4.0/. The Creative Commons Public Domain Dedication waiver (http://creativeco mmons.org/publicdomain/zero/1.0/) applies to the data made available in this article, unless otherwise stated in a credit line to the data. 
The SORL1 gene (Sortilin-related receptor 1) encodes a $\sim 250-k D a$ transmembrane protein, termed Sortilin-related receptor (SorLA), with multiple functional domains. The ectodomain of SorLA is a complex mosaic structure comprising a Vps10p domain, LDLR class B and class A repeats, an EGF-like domain, and a cassette of six fibronectin type-3 domains (Fig. 1a). Newly synthesized SorLA molecules follow the constitutive secretory pathway from the endoplasmic reticulum (ER) to the cell surface, through the Golgi. During their transport through the secretory pathway, SorLA proteins are subjected to several post-translational modifications, including both $\mathrm{N}$ - and $\mathrm{O}$-glycosylation, and maturation processes [3-9], resulting in the presence of immature and mature species. At the cell surface, SorLA molecules can be subject to proteolytic shedding, releasing the soluble ectodomain of the receptor, termed soluble SorLA (sSorLA) $[8,10]$. Intact SorLA molecules undergo clathrin-dependent endocytosis [11]. Internalized molecules move to the early endosomes from where most of them will sort to the trans-Golgi network (TGN) to continuously shuttle between TGN and endosomes thereafter (reviewed in [12]). As alternative routes, SorLA may sort from endosomes to the cell surface [13] or to lysosomes [14].

Several studies have highlighted a protective function of wild-type SorLA against AD. Indeed, loss of SORL1 expression in several mouse models of $\mathrm{AD}$ increases extracellular $\mathrm{A} \beta$ levels, the aggregation of which triggers AD pathology [15-17]. On the other hand, overexpression of SORL1 in neuronal and non-neuronal cell lines limits the processing of APP, the A $\beta$ precursor protein, and decreases $A \beta$ production $[5,15,18,19]$. The known mechanisms whereby SorLA acts to lower $A \beta$ production have been reviewed recently in details $[12,20]$. First, SorLA is involved in the trafficking and recycling of APP. By binding to the retromer complex, SorLA redirects APP from the early endosomes back to the Golgi apparatus through the recycling pathway. This in turn results to reduced amyloidogenic processing of APP, by preventing it from being targeted to the late endosome compartments, where APP is cleaved into A $\beta$ by the $\beta$ - and $\gamma$-secretases. Second, SorLA may directly bind nascent $\mathrm{A} \beta$ peptides and target them to lysosomes, which also contributes to the reduction of $A \beta$ secretion [21].

SORL1 is now considered as a major AD risk factor gene, because of increased frequencies of rare coding variant carriers in cases as compared to cognitively-healthy controls [2, 22-24]. The association has been demonstrated at the gene level. Indeed, aggregating protein-truncating SORL1 variants (PTV, all of which are ultra-rare) in cases and controls showed odds ratios ranging from $\sim 12$ to $\sim 28$, among all $\mathrm{AD}$ and EOAD cases, respectively [24]. This suggests a strong effect and thus a putative clinical utility. However, the penetrance of such variants appears to be incomplete, so that they should still be considered with caution in a clinical setting [25]. In terms of variant clinical interpretation, as most PTVs lead to reduced SorLA levels through nonsense-mediated decay (NMD) $[22,23,26]$ and/or to the putative production of truncated proteins and hence increased $A \beta$ production, one could consider that most PTVs are likely strong $\mathrm{AD}$ risk factors in case they are to be used in a clinical setting. Regarding missense variants, however, variant interpretation may not be as straightforward. Evidence of an association also relies on burden tests, aggregating rare (allele frequency below 1\%), missense SORL1 variants, following their selection based on in silico deleteriousness predictions. Gene-level odds ratio are very heterogeneous, depending on the selection criteria for missense variants based on more or less stringent allele frequency and deleteriousness prediction thresholds [24, 27]. Due to their extreme rarity and diversity, it remains impossible to assess the association of rare SORL1 missense variants with $\mathrm{AD}$ at the variant level. In addition, contrary to PTVs, the functional effect of missense variants and their pathophysiological mechanisms are hard to predict. Whether or not a given missense variant behaves as a loss-of-function variant and by which mechanisms remain to be determined for most of them. Hence, most SORL1 missense variants can basically not be used in a clinical setting, up to now. To date, only 6 missense variants have been studied in vitro and have shown varying

(See figure on next page.)

Fig. 1 Identification of SorLA missense variants that reduce production of the complex-glycosylated mature form of the protein in HEK293 cells. a For the schematic presentation of SorLA domains structure, we used the domains provided by Uniprot (https://www.uniprot.org/uniprot/Q92673): signal peptide (a.a. 1-28), propeptide (a.a. 29-81), Vps10p (BNR repeats) (a.a. 136-752), LDLR class B repeats (a.a. 800-1013), EGF-like (a.a. 10261072), LDLR class A repeats (a.a. 1076-1551), Fibronectin type III-cluster (a.a. 1557-2118), Transmembrane domain (a.a. 2138-2158) and Cytoplasmic tail (a.a. 2159-2214). The Mis3, Mis2, and Mis0-1 missense variants examined in this study are shown in red, orange and black, respectively. The 15 Mis3 variants presenting maturation defects are placed on the left of the schematic presentation of the SorLA protein. $\mathbf{b}$ Western Blot analyses of the SorLA protein maturation profile in HEK293 cells transiently expressing wild-type (WT) and missense variants of SorLA. For each variant, the pattern was confirmed in at least 2 independent replicates. Blots were probed with an anti-SorLA antibody and representative blots are presented. Immature core-glycosylated and mature complex-glycosylated SorLA are indicated with solid and empty arrowheads, respectively. The molecular masses of marker proteins in $\mathrm{kDa}$ are shown on the left. Maturation-deficient SorLA variants are highlighted with a red rectangle 


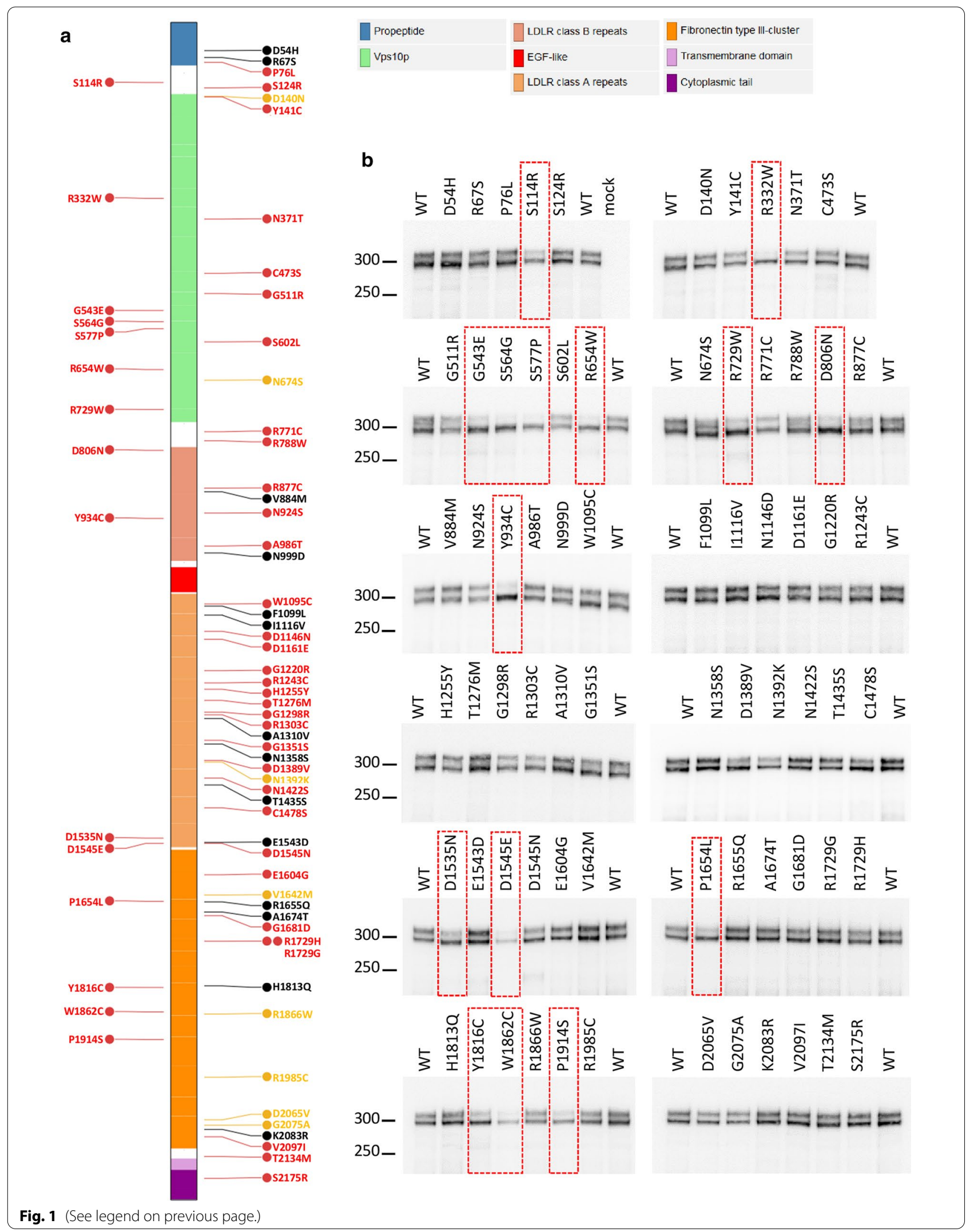


degrees of decrease in SorLA protein function [21, 28, 29]. Among them, demonstration of an associated mechanism was shown for only one, p.Gly511Arg, which diminished the ability of mutant SorLA to target nascent $A \beta$ peptides to lysosomes. Other missense variants might act by reducing SorLA binding to other partners, including APP, or by resulting in an unstable, misfolded or immature protein.

Here, we analyzed 70 rare missense SORL1 variants. An initial screening, based on overexpression in HEK293 cells, revealed that 15 of them showed protein maturation and plasma membrane targeting defects. Three of these variants and two maturation-competent variants were further studied in details on endogenous SORL1 in CRISPR/Cas9-modified hiPSCs, validating the results obtained in HEK293 cells and highlighting gradual effects of the variants. We also demonstrated that two variants affecting the proper maturation of SorLA were associated with an increase of $A \beta$ secretion in the cellular medium, thus providing a direct link with $\mathrm{AD}$ pathophysiology. Finally, structural analysis revealed that this impaired cellular trafficking could result from subtle variations of the protein 3D structure and changes in the interatomic interactions.

\section{Materials and methods \\ Selection of variants}

SORL1 rare missense variants were selected among a series of 1383 exomes of unrelated probands with AD (EOAD in $97 \%$ of cases) from the national reference center for young Alzheimer patients (CNRMAJ, Rouen, France). Inclusion criteria and exome sequencing procedures and QC are described in details in our previous reports [2, 22, 27, 30]. Briefly, patients fulfilled the diagnosis of probable or definite AD [31]. Diagnoses were based on clinical examination by a neurologist or geriatrician from French Memory Clinics and included personal medical and family history assessments, neurological examination, neuropsychological assessment, and neuroimaging. In addition, cerebrospinal fluid biomarkers indicative of $\mathrm{AD}$ were available for $80 \%$ of the cases who were selected for whole exome sequencing (lumbar puncture not perfomed in the $20 \%$ remaining). None of the patients carried a likely pathogenic variant in a Mendelian dementia gene [30]. From exome sequencing data, after $\mathrm{QC}$, we selected rare (allele frequency $<1 \%$ in the gnomAD non neuro, database v2.1 [32]) missense SORL1 variants and classified them into Mis 0,12 or 3 categories depending on the number of bioinformatics tools predicting the variant to be deleterious or not among Polyphen-2, SIFT and Mutation Taster. For inclusion in this study, we considered all rare Mis3 variants and selected some $<$ Mis3 variants (Additional file 3: Table S1). In addition, we selected 8 variants identified in previous studies ([2, 27], including two Mis3 singletons found in controls, ending up with a total of 70 variants distributed all along the SorLA protein coding sequence.

\section{cDNA constructs}

The SorLA $A^{759}$ and SorLA ${ }^{2131}$ constructs were designed following the constructs described in [3]. The wild-type human SorLA ${ }^{759}$ (Met ${ }^{1}$-Glu ${ }^{759}$ ) cDNA construct was generated by PCR-amplification on the pCMV6-XL5human full-length SORL1 cDNA plasmid (pCMV6-XL5WT-SorLA ${ }^{\mathrm{FL}}$ OriGene Technologies, Inc, Rockville, MD, USA) using the $5^{\prime}$ CCGGAATTCCGGCAAAATGGC GACACGGAGCAGCAGG $3^{\prime}$ and $5^{\prime}$ TGCTCTAGA GCACTACTCGTTCTCTTCTGCCAGGGG $3^{\prime}$ oligonucleotides. Residue numbers refer to the primary structure of the wild-type protein. The SorLA ${ }^{759}$ PCR products were next subcloned as an EcoRI/Xba fragment into the pCMV6-XL5 expression vector. The wild-type human SorLA ${ }^{2131}$ (Met ${ }^{1}$ - $\mathrm{Ala}^{2131} / \mathrm{Val}^{2209}-\mathrm{Ala}^{2214}$ ) cDNA construct was generated using the GeneArt ${ }^{\mathrm{TM}}$ Site-Directed Mutagenesis System (Invitrogen ${ }^{\mathrm{TM}}$, Carlsbad, CA, USA) and a PCR overlap extension procedure on the pCMV6XL5-human full-length SORL1 cDNA plasmid. This construct comprises the cDNA sequence encoding the luminal part of SorLA (amino acids 1-2131), fused to the cDNA sequence encoding the last 6 amino acids of the protein (amino acids 2209-2214). The sequences of mutagenized oligonucleotides were: 5' GCA TCT GCA ACG CAG GCT GCC GTC CCC ATG GTG ATA GCC TGA AAG AGC $3^{\prime}$ and $5^{\prime}$ GCT CTT TCA GGC TAT CAC CAT GGG GAC GGC AGC CTG CGT TGC AGA TGC $3^{\prime}$ s. Integrity of the SorLA ${ }^{759}$ and SorLA ${ }^{2131}$ cDNA constructs were verified by sequencing. Site-directed mutagenesis was then carried out over the pCMV6-XL5WT-SorLA $^{\mathrm{FL}}$, pCMV6-XL5-WT-SorLA ${ }^{759}$ or pCMV6XL5-WT-SorLA ${ }^{2131}$ plasmids using the QuikChange II XL Site-Directed Mutagenesis Kit from Agilent (Santa Clara, CA, USA) according to manufacturer's instructions. All oligonucleotides used are listed in Additional file 4: Table S2. Integration of the mutation was verified by sequencing. The plasmidic constructs with S114R, R332W, G543E, S564G, S577P, R654W, R729W, D806N, Y934C, D1535N, D1545E, P1654L, Y1816C, W1862C, P1914S SorLA variants were sequenced in their entirety.

\section{HEK293 cells culture and transfection}

HEK293 cells were grown and maintained in DMEM/ F12 medium (Gibco/Thermo Fisher Scientific, Waltham, MA, USA) supplemented with 10\% FCS (Eurobio, Les Ulis, France). Cells were plated in 12-well or 6-well plates $48 \mathrm{~h}$ prior to transfection, grown to approximately $90 \%$ 
confluence and transfected with the appropriate amounts of the indicated constructs using the lipofectamine 3000 reagent (Invitrogen ${ }^{\mathrm{TM}}$ ) according to the manufacturer's protocol. Twenty-four or $48 \mathrm{~h}$ after transfection, the cells were harvested and processed for Western blotting or biotinylation analyses.

\section{hiPSC culture}

The hiPS cell line was previously generated by our group. This cell line derives from an unaffected male donor and has an APOE 3/3 genotype. The line was analyzed for pluripotency markers expression by quantitative PCR and for chromosomal abnormalities by karyotyping, and was able to differentiate into the three germ layers. Whole exome sequencing of the parental hiPSC line confirmed the absence of rare coding variants in the SORL1 gene, as well as in APP, PSEN1, PSEN2, TREM2, ABCA7 and other genes causing Mendelian forms of dementia [30]. hiPSCs were cultured on feeder-free conditions in mTeSR Plus medium (STEMCELL Technologies, Vancouver, Canada) on Matrigel-coated culture dishes (Corning, Corning, NY, USA) diluted in DMEM-F12 according to manufacturer's instructions in a $37^{\circ} \mathrm{C} / 5 \% \mathrm{CO}_{2}$ incubator. Cells were split when they reached $80 \%$ confluency using StemPro Accutase (Thermo Fisher Scientific) and plated in $10 \mu \mathrm{M}$ ROCK inhibitor (StemGent, Cambridge, MA, USA) supplemented medium. Medium was refreshed the next day to remove ROCK inhibitor. Differentiating colonies were removed from the plate before splitting. Cell lines were confirmed to be free of mycoplasma.

\section{CRISPR/Cas9 genome editing}

Genome editing was performed following a published protocol [33]. The guide RNAs (gRNAs) were designed using the CRISPOR.org web tool [34] (http://crispor.tefor. net/). Two gRNAs were designed for each variant. We chose guide sequences located as close to the mutation site, with the highest specificity score (Additional file 5: Table S3). All gRNAs were cloned into the $\mathrm{pX} 458$ vector (Addgene, pSpCas9(BB)-2A-GFP, plasmid \#48,138) that coexpresses the Cas9 nuclease and the GFP. Each plasmid and its corresponding single-stranded oligodeoxynucleotide (ssODN) were nucleofected in the hiPSC line, using an AMAXA nucleofector II device. Two days later, cells were stained using DAPI (NucBlue ${ }^{\mathrm{TM}}$ Fixed Cell ReadyProbes reagent, Molecular Probes, Thermo Fisher Scientific) as viability dye. GFP-positive cells among living cells were sorted and plated as 1 cell/well into 96-well plates using FACS Aria III (BD Bioscience, Franklin Lakes, NJ, USA). When clones reached $80 \%$ confluency, genomic DNA was isolated and analysed by Sanger sequencing (PCR primers are listed in Additional file 5: Table S3). For each variant, 6 clones were selected, including three clones carrying the variant at the homozygous state and three isogenic wild-type clones. For SORL1-KO clone selection, we selected clones carrying a homozygous frameshift insertion. The absence of the protein was then verified using Western blotting (Additional file 1: Fig S5).

\section{Biotinylation}

Subconfluent cultures were rinsed twice with $\mathrm{PBS}+/+$, exposed to sulfo-NHS-SS-biotin at $1 \mathrm{mg} / \mathrm{mL}$ in PBS $+1+$ (Thermo Fisher Scientific) for $30 \mathrm{~min}$ at $4^{\circ} \mathrm{C}$, rinsed three times with glycine quenching buffer (100 mM glycine in PBS $+/+$ ) and solubilized in RIPA buffer $(50 \mathrm{mM}$ Tris/HCl pH8, $150 \mathrm{mM} \mathrm{NaCl}, 0.5 \%$ (w/v) deoxycholic acid, 1\% NP40, 10\% glycerol, 2 mM DTT), supplemented with a cocktail of protease inhibitors (Sigma-Aldrich) and phosphatase inhibitors (Halt phosphatase, Thermo Fisher Scientific) for $10 \mathrm{~min}$ on ice. The resulting lysate was centrifuged at $12,000 \times g$ for $10 \mathrm{~min}$ at $4^{\circ} \mathrm{C}$, and total cellular protein content was determined using the DC Protein Assay Kit (Bio-Rad Laboratories, Hercules, CA, USA). Then, $200 \mu \mathrm{g}$ of proteins were incubated with streptavidin beads (Thermo Fisher Scientific) for $1 \mathrm{~h}$ at $4^{\circ} \mathrm{C}(4 \mu \mathrm{g}$ of protein/ $\mu \mathrm{l}$ of beads pre-equilibrated with RIPA buffer). The supernatant was removed using a magnetic stand, then beads were washed five times with RIPA buffer, and resuspended with $1 \times$ Laemmli sample buffer (Bio-Rad Laboratories) containing 100 mM DTT.

\section{Protein extraction}

Cells were homogenized in RIPA buffer (Tris- $\mathrm{HCl} \mathrm{pH} 8$ $0.05 \mathrm{M}, \mathrm{NaCl} 0.15 \mathrm{M}, \mathrm{NP}-40$ 1\%, Sodium deoxycholate $0.5 \%$, Glycerol $10 \%$, DTT $2 \mathrm{mM}$ ), supplemented with a cocktail of protease inhibitors (Sigma-Aldrich, SaintLouis, MI, USA) and phosphatase inhibitors (Halt phosphatase, Thermo Fisher Scientific). After $10 \mathrm{~min}$ on ice, lysates were centrifuged $\left(12,000 \times \mathrm{g}, 10 \mathrm{~min}, 4^{\circ} \mathrm{C}\right)$ and the supernatant containing soluble proteins was collected. Protein concentrations were measured using the DC Protein Assay Kit (Bio-Rad Laboratories).

\section{Glycosidase treatments}

PNGase F, O-glycosidase, Neuraminidase and Endo $\mathrm{H}$ were obtained from New England Biolabs (Ipswich, MA, USA). Digestions were performed according to the manufacturer's instructions. All digestions were carried out for $24 \mathrm{~h}$ at $37^{\circ} \mathrm{C}$ for PNGase F/O-glycosidase/ neuraminidase treatments and for $3 \mathrm{~h}$ for Endo $\mathrm{H}$ treatments. After this, the proteins were analyzed by SDS-PAGE as below.

\section{Western blotting}

Proteins were resolved by Tris-acetate NOVEX NuPAGE 3-8\% (Invitrogen) (SorLA ${ }^{\mathrm{FL}}$ and SorLA ${ }^{2131}$ ) or TGX Stain-Free gels (Bio-Rad Laboratories) (SorLA $^{759}$ and 
samples from biotinylation experiments) then transferred onto nitrocellulose membrane using the Trans-Blot Turbo system (Bio-Rad Laboratories). Membranes were then blocked in 5\% non-fat milk and immunoblotted with the appropriate primary antibody: mouse monoclonal anti-SorLA (1:5,000; mAb\# 611,860, BD Bioscience) for the detection of full length SorLA and SorLA ${ }^{2131}$ proteins; rabbit monoclonal anti-SorLA antibody specific to the N-terminus of human SorLA protein (1:1,000; D8D4G \#79,322; Cell Signaling, Danvers, MA, USA) for the detection of SorLA ${ }^{759}$ proteins; rabbit polyclonal antiFUS (1:5,000; A300-302A; Bethyl Laboratories, Montgomery, TX, USA). Membranes were then incubated with secondary peroxidase-labelled anti-mouse or antirabbit antibodies $(1: 10,000)$ from Jackson Immunoresearch Laboratories (WestGrove, PA, USA), and signals were detected with chemiluminescence reagents (ECL Clarity, Bio-Rad Laboratories). Signals were acquired with a GBOX (Syngene, Cambridge, UK), monitored by the Gene Snap software (Syngene). When appropriate, the signal intensity in each lane was quantified using the Genetools software (Syngene) and normalized with the Stain-Free signal quantified in the corresponding lane (ImageLab $^{\mathrm{TM}}$ software, Bio-Rad Laboratories).

\section{$A \beta$ secretion assay}

hiPSCs were plated on matrigel pre-coated 12-well plates in triplicate. Two days later, medium was replaced by $500 \mu \mathrm{L}$ of mTeSR Plus supplemented with phosphoramidon (10 $\mu \mathrm{M}$, Sigma-Aldrich). Twenty-four hours later, conditioned medium was collected in each well and centrifuged at $1000 \times g$ for $5 \mathrm{~min}$. The supernatant was supplemented with a cocktail of protease inhibitors (Sigma-Aldrich) and frozen at $-80{ }^{\circ} \mathrm{C}$ until use. The quantification of $\mathrm{A} \beta$ peptides was performed using the MSD technology (Meso Scale Diagnostics, Rockville, MD, USA) with the V-PLEX A $\beta$ Peptide Panel 1 (6E10) kit according to manufacturer's instructions. Conditioned media were diluted $1: 2$ in Diluent 35 and analyzed in duplicate. The plate was analyzed using a MESO QuickPlex SQ 120 instrument (Meso Scale Diagnostics). $A \beta$ quantities were normalized to the total protein amount measured in each sample.

\section{Immunofluorescence}

hiPSCs were plated on matrigel-coated $20 \mathrm{~mm}$ round coverslips. Twenty-four hours later, cells were rinsed twice with PBS before fixation with $1 \mathrm{ml}$ of $4 \%$ paraformaldehyde for $1 \mathrm{~h}$ at room temperature followed by three washes in PBS of 10 min each. Antigen retrieval was performed according to Hayashi et al. [35]. Briefly, coverslips were immersed in a $6 \mathrm{M}$ urea-0.1 M Tris (pH9.5) bath at $80^{\circ} \mathrm{C}$ for $10 \mathrm{~min}$, followed by three washes in PBS of 15 min each. Cells were permeabilized with PBS/0.1\% Triton X-100 for $5 \mathrm{~min}$, and blocked for $30 \mathrm{~min}$ in antibody buffer [PBS containing $2 \%$ bovine serum albumin (BSA) and 3\% normal goat serum (NGS)]. Cells were then incubated with primary antibodies in antibody buffer for $1 \mathrm{~h}$ at room temperature. Following three washes with PBS (10 min each), cells were incubated with secondary antibodies in antibody buffer at room temperature for $1 \mathrm{~h}$ in the dark. Following two washes with PBS (10 min each), cells were counterstained with DAPI (NucBlue $^{\mathrm{TM}}$ Fixed Cell ReadyProbes reagent, Molecular Probes, Thermo Fisher Scientific) and coverslips were finally mounted in ProLongTM Diamond antifade solution (Thermo Fisher Scientific). The following antibodies were used: anti-SorLA 48/LR11 (1:200; 612,633; BD Biosciences), anti-Rab5 (1:250; ab218624; Abcam, Cambridge, UK), anti-Cyclophilin B (1:500; ab16045; Abcam), goat anti-mouse IgG Alexa Fluor (1:600; Molecular probes, Thermo Fisher Scientific), goat anti-rabbit IgG Alexa Fluor (1:600; Molecular Probes, Thermo Fisher Scientific). Images were acquired using a Leica THUNDER Imaging System (Leica Microsystems, Wetzlar, Germany), combining Computational Clearing with Large Volume adaptative deconvolution (LVCC) for post-acquisition processing. Brightness and contrast of images were adjusted using the Fiji software [36].

\section{Co-localization analysis}

Co-localization analysis of SorLA with endoplasmic reticulum and early endosomes markers was performed using JACoP plugin [37] on Fiji environment (RRID:SCR_002285) and presented as M1 Manders' correlation coefficients. The Manders' M1 coefficients indicate the proportion of SorLA (green channel) coincident with Rab5 or Cyclophilin B (red channel) over its total intensity.

\section{Structural analysis}

The structure of the Vps10p domain of SorLA in complex with its own propeptide fragment (PDB ID 3WSY) was used as a model for structural analysis [38]. The changes of stability and flexibility of the Vps10p domain upon mutation were estimated with the DynaMut webserver [39] (http://biosig.unimelb.edu.au/dynamut/). The structures were analyzed using PyMOL (The PyMOL Molecular Graphics System, Schrodinger, LLC) or UCSF Chimera [40] softwares.

\section{Statistical analysis}

For each experiment, we compared the variants to the WT forms of SorLA by performing a regression model. Variants were all considered into one independent factor 
where the WT was the reference level. In Western blot experiments, the outcome was the normalized signal intensity, in $\log$ scale to overcome heteroscedasticity whereas in $A \beta$ assay, the outcome was the normalized quantification of $A \beta$ peptide. Models were all adjusted for experiment settings. For each variant, the p-value refers to the nullity test of its associated coefficient in the model. We adjusted p-values for multiple testing using the Bonferroni's correction. Statistical models were performed using the R software. All tests were two-tailed at the significance level of $5 \%$ (ns: not significant, " $p<0.05$, *** $\left.p<0.01,{ }^{* * * * *} p<0.001\right)$.

\section{Results}

Identification of SorLA variants that reduce the level of complex-glycosylated mature SorLA protein in HEK293 cells

We studied 70 rare missense variants, distributed all along the SorLA protein (Fig. 1a) and classified according to their predicted deleteriousness assessed by three bioinformatic tools: PolyPhen-2, MutationTaster and SIFT. Forty-eight of them were predicted damaging by all three bioinformatics tools (Mis3 variants), 8 were predicted damaging by two of the three tools (Mis2) and 14 were predicted damaging by one or none of the tools (Mis10) (Fig. 1a). To study the effect of these rare SORL1 missense mutations on SorLA protein maturation, cDNA constructs encoding the full-length wild-type (WT)SorLA or SorLA variants were used to transfect HEK293 cells for transient expression. When overexpressed in HEK293 cells, WT-SorLA protein consisted of two distinct forms on immunoblot analysis (Fig. 1b, Fig. 2) that were hypothesized to be different glycoforms of the protein. As previously shown $[3,9]$, removal of all $N$-linked glycans by treatment with PNGase F resulted in a single band that migrates at $\sim 250 \mathrm{kDa}$, which corresponds to the predicted molecular weight of the full-length SorLA $\left(\right.$ SorLA $^{\mathrm{FL}}$ ) protein (Fig. 2a). Treatment with neuraminidase altered the mobility of only the upper band that subsequently migrated as the lower form, indicating that the upper species correspond to complex type $N$-glycans capped with sialic acid residues (Fig. 2a). No further shift in the migration was seen while combining neuraminidase and O-glycosidase (Fig. 2a), or O-glycosidase and PNGase F (Fig. 2b), suggesting that SorLA lacks O-glycosylation in HEK293 cells. The conversion of highmannose type $N$-glycans to complex $N$-glycans occurs in the medial Golgi region. When proteins are correctly processed through the endoplasmic reticulum (ER) and Golgi, $N$-glycans become resistant to Endo H. Sensitivity to Endo $\mathrm{H}$ therefore indicates the presence of proteins bearing high mannose $N$-glycans that still reside in the ER. In our experiments, Endo $\mathrm{H}$ digestion yielded two bands with reduced molecular size (Fig. 2c). As expected, the upper band that corresponds to mature complex type $N$-glycans capped with sialic acid residues, was somewhat resistant to Endo H. However, the lower SorLA species were equally sensitive to treatment with Endo $\mathrm{H}$ and PNGase F yielding a band at $\sim 250 \mathrm{kDa}$, indicating that they correspond to species bearing high mannose $N$-glycans. Together, these data indicated that SorLA exists as two species in HEK293 cells: a mature complex-glycosylated form of SorLA that corresponds to the upper band of the doublet and an immature oligomannoside $\mathrm{N}$-linked core-glycosylated form of the protein that corresponds to the lower band.

Of the 70 missense variants studied, 55 displayed a maturation profile similar to the one observed for WTSorLA (Fig. 1b). In contrast, 15/70 missense variants (15/48 of Mis3 variants $(\sim 30 \%))$, showed a drastic reduction of the upper band corresponding to the glycoform of the protein bearing complex-type $N$-glycans (S114R, R332W, G543E, S564G, S577P, R654W, R729W, D806N, Y934C, D1535N, D1545E, P1654L, Y1816C, W1862C, and P1914S) (Additional file 1: Fig. S1), indicating defects in the maturation of complex $N$-glycans for these variants. These data are summarized in Additional file 3: Table S1.

\section{SorLA variants associated with abnormal immunoblot profiles interfere with SorLA transport to the plasma membrane in HEK293 cells}

The reduced level of fully mature SorLA could be associated with transport defects of the protein along the secretory pathway, thereby preventing SorLA delivery to the cell surface. To explore the impact of SORL1 variants on SorLA transport along the secretory pathway, we took advantage of constructs leading to secreted truncated forms of SorLA, comprising different parts of the luminal part of SorLA, but not its cytoplasmic tail or transmembrane segment. These constructs encoded either amino acids $1-759$ (SorLA ${ }^{759}$ ) or amino acids 1-2131 (SorLA ${ }^{2131}$ ) of the SorLA protein (Fig. 3a) [3]. After transient transfection in HEK293 cells, intracellular or secreted WT-SorLA proteins were subjected to Western blotting. The WT-SorLA ${ }^{759}$ and WT-SorLA ${ }^{2131}$ proteins were detected both in the cellular lysate and in the medium (Fig. 3b, Additional file 1: Fig. S2), indicating that both truncated constructs reached the plasma membrane and were efficiently secreted. Treatment of WT SorLA ${ }^{2131}$ and SorLA ${ }^{759}$ proteins with glycosidase showed that the species present in the lysate were immature high-mannose type $\mathrm{N}$-glycans (PNGase F and Endo $\mathrm{H}$ sensitive, Neu insensitive), whereas the secreted forms of the protein corresponded to mature complex $N$-linked 


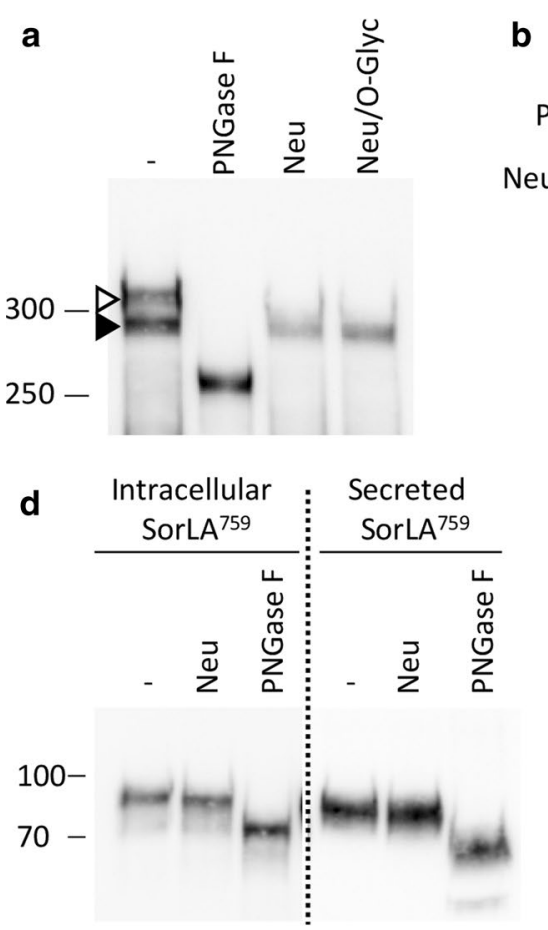

b

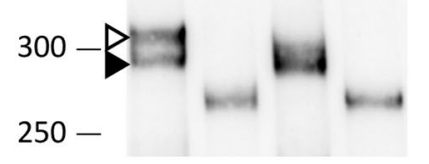

e

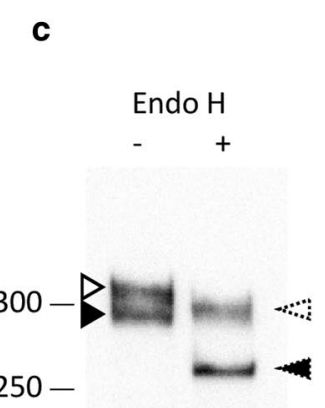

$250-$
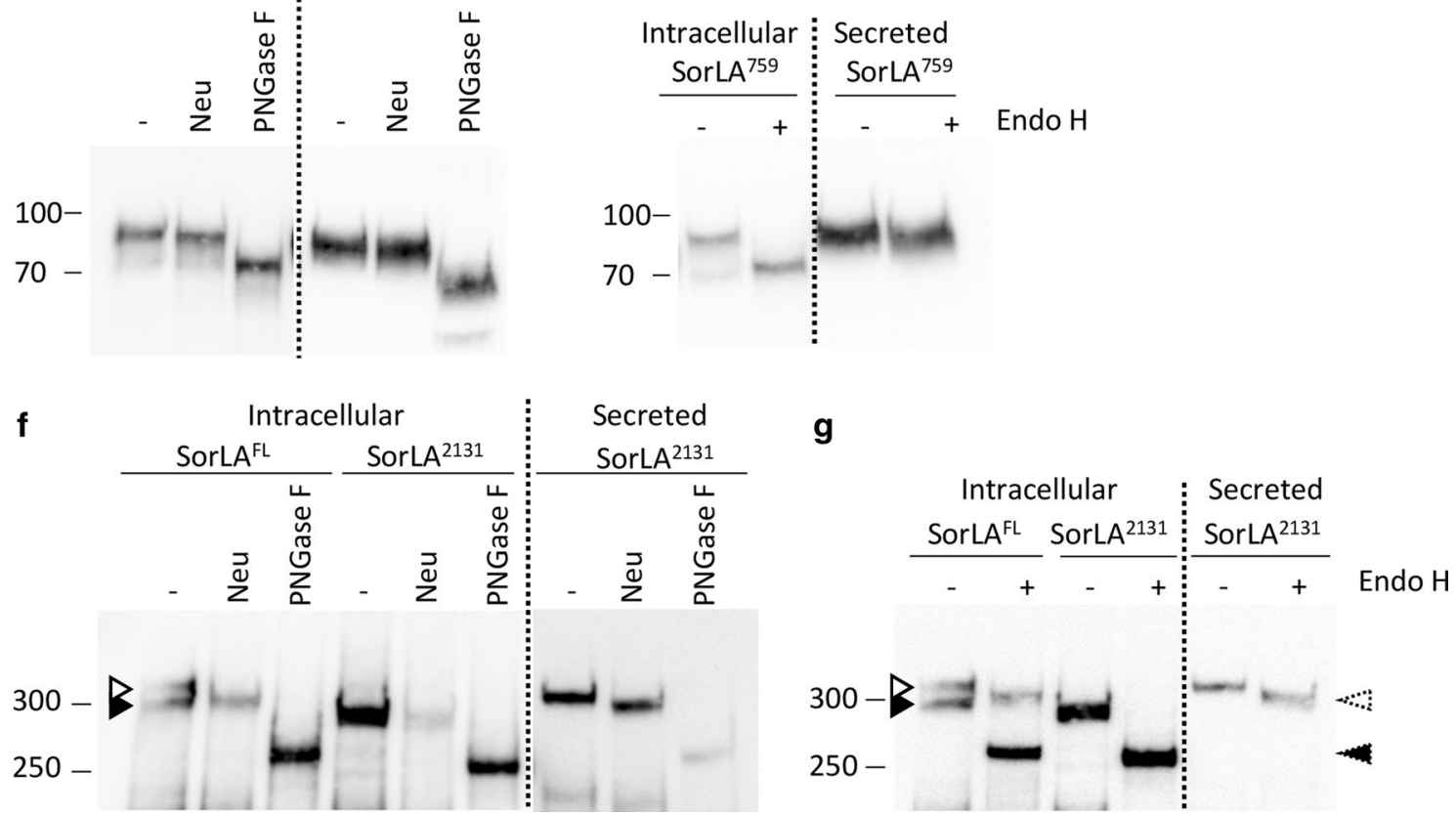

Fig. 2 Characterization of the maturation process of SorLA proteins in HEK293 cells. Western blot analyses after glycosidase treatments of wild-type full-length (SorLA ${ }^{\mathrm{FL}}$ ) $(\mathrm{a}, \mathrm{b})$ or secreted truncated (SorLA ${ }^{759}$ or SorLA $\left.{ }^{2131}\right)(\mathrm{c}-\mathrm{g})$ forms of SorLA proteins transiently overexpressed in HEK293 cells. Blots were probed with an anti-SorLA antibody and representative blots are presented. Cellular lysates (intracellular) or supernatants (secreted) were treated in the absence or presence of the glycosidases PNGase F, Neuraminidase (Neu), O-Gycosidase (O-Glyc). Untreated samples (-) were incubated under the same conditions but without enzymes. PNGase F cleaves both high-mannose, hybrid and complex $N$-glycans from glycoproteins. Endo $\mathrm{H}$ cleaves preferentially high-mannose type $\mathrm{N}$-glycans, but not complex $\mathrm{N}$-glycans. Neuraminidase cleaves sialic acid from either complex or O-glycans. O-glycosidase removes O-linked glycans, but this cleavage is more effective only after removing terminal sialic residues by a neuraminidase. The positions of immature core-glycosylated and mature complex-glycosylated forms of SorLA are indicated with solid and empty arrowheads, respectively. Resulting corresponding species obtained after neuraminidase treatment are indicated with dashed arrowheads. The molecular masses of marker proteins in $\mathrm{kDa}$ are shown on the left

glycoproteins (PNGase F and Neu sensitive, Endo H partially resistant) (Fig. 2d-g).

The 15 missense variants with the more drastic maturation-defective effect in the context of the full-length protein (S114R, R332W, G543E, S564G, S577P, R654W, R729W, D806N, Y934C, D1535N, D1545E, P1654L, Y1816C, W1862C, and P1914S) and 12 missense variants presenting a wild-type maturation profile (P76L, S124R,
D140N, Y141C, N371T, C473S, G511R, S602L, E1543D, E1604G, G1681D, and R1729G) were introduced into the WT-SorLA ${ }^{759}$ and WT-SorLA ${ }^{2131}$ constructs by sitedirected mutagenesis (Fig. 3a). All assessed SorLA variants were detected in the cellular lysate, indicating that they were properly expressed in HEK293 cells (Additional file 1: Fig. S2). The truncated constructs carrying the S114R, R332W, G543E, S564G, S577P, R654W, 
D806N, Y934C, D1535N, D1545E, W1862C or P1914S variant, associated with a deficient maturation profile using the full-length construct, all showed a statistically significant lower level of secretion compared to their corresponding wild-type construct (Fig. 3b, 3c, Additional file 3: Table S1, Additional file 7: S4). The R729W variant also resulted in a lower level of secretion, but did not reach statistical significance. The P1654L and Y1816C variants did not show statistically significant difference compared to the WT-SorLA ${ }^{2131}$. Of the 13 missense variants that displayed a wild-type maturation profile (P76L, S124R, D140N, C473S, G511R, S602L, E1543D, E1604G, G1681D, R1729G), all presented a level of secretion similar to the wild-type form of their matching constructs. Note that some variants (S124R, R332W, G511R) have been introduced both in the SorLA ${ }^{759}$ and SorLA ${ }^{2131}$ constructs and yielded similar quantitative results. The results obtained using secreted truncated forms of SorLA suggest that maturation defects of glycosylation are associated with a default of protein transport towards the plasma membrane.

To validate these results in the context of the full-length SorLA protein, we evaluated the amount of SorLA at the plasma membrane by cell-surface biotinylation experiments (Fig. 4). Compared to the wild-type form of the protein, all SorLA variants exhibiting maturation defects (S114R, R332W, G543E, S564G, S577P, R654W, R729W, D806N, Y934C, D1535N, D1545E, P1654L, Y1816C, W1862C, P1914S) showed a lower amount of biotinylatable cell-surface SorLA, with a less drastic effect for the variants located in the Fibronectin-type III domain. The cytosolic FUS protein was used as a control to exclude non-specific labelling of the intracellular fractions. As expected, no FUS staining was detected in the biotinylated fraction.

Altogether, these data show that maturation defects are associated with an impairment of SorLA delivery to the cell surface.

\section{SorLA variants interfere with SorLA maturation} and transport to the plasma membrane in hiPSC

To validate the functional effect of rare missense variants in the context of endogenous SORL1 expression, we used CRISPR/Cas9 gene editing to introduce three maturation-defective variants (R332W, S577P and R654W) and two variants showing a wild-type maturation profile (S124R and N371T) into a control hiPSC line previously generated by our group. Sanger sequencing of colonies derived from single putatively edited cells confirmed the presence of the S124R, R332W, N371T, S577P and R654W variants at the homozygous state in each respective cell line (Additional file 1: Fig. S3).

We first assessed SorLA maturation profile in CRISPR/ Cas9-edited hiPSC and isogenic wild-type control cells by Western blot analyses. In the wild-type cell lines, the SorLA protein displayed a two-band migration profile, with the upper band being the overwhelming majority (Fig. 5a). Glycosidase treatment confirmed that the upper band corresponded to mature complex N-linked glycoproteins (PNGase F and Neu sensitive, Endo $\mathrm{H}$ partially resistant), and the lower band to immature high-mannose type $\mathrm{N}$-glycans (PNGase $\mathrm{F}$ and Endo $\mathrm{H}$ sensitive, Neu insensitive) (Fig. 5a). The S124R and N371T hiPSC lines showed a SorLA migration profile similar to that observed for the wild-type cell lines (Fig. 5b). On the other hand, in the R654W hiPSC lines, we observed a drastic downwards mobility shift of the SorLA proteins. Regarding the R332W and the S577P clones, we detected an intermediate profile, with a decrease in the intensity of the upper band and concomitantly an increase in the intensity of the lower band, appearing to be more pronounced for the R332W line (Fig. 5b). These patterns were confirmed in three independent hiPSC clones, indicating that the results were not clone-dependent.

In HEK293 cells, altered maturation profile correlated to defects in SorLA targeting to the plasma membrane, and lower SorLA levels at the plasma membrane. We thus performed cell-surface biotinylation experiments on wild-type and CRISPR/Cas9-modified hiPSCs. In wild-type cells, only the mature band was present at the

\footnotetext{
(See figure on next page.)

Fig. 3 SorLA variants associated with abnormal immunoblot profiles interfere with the secretion of SorLA truncated constructs in HEK293 cells. a Schematic representation of the soluble truncated SorLA ${ }^{759}$ and SorLA ${ }^{2131}$ proteins, and the location of SorLA variants examined. The Mis3, Mis2, and Mis0-1 missense variants studied are shown in red, orange and black, respectively. Maturation-deficient SorLA variants are underlined. $\mathbf{b}$ Wild-type (WT) and mutants SorLA proteins were expressed in HEK293 cells. The SorLA proteins secreted into the cellular medium were analyzed by immunoblotting using an anti-SorLA antibody. Total protein was used as the loading control with Stain-free (SF) technology. Representative blots are presented. Maturation-deficient SorLA variants are underlined. The molecular masses of marker proteins in kDa are shown on the left. c Quantification of secretion efficiency from 4 independent experiments (SorLA ${ }^{759}$ ) or 3 independent experiments $\left(\right.$ SorLA ${ }^{2131}$ ). The normalized quantification of the SorLA proteins in the cellular medium is reported in the graphs. For both SorLA ${ }^{759}$ and SorLA ${ }^{2131}$, WT-SorLA were arbitrarily set at 100 arbitrary units. Protein levels were compared by using a regression model adjusted for experiment as random effect. P-value significances are displayed after Bonferroni's correction for 14 and 17 statistical tests for SorLA ${ }^{759}$ and SorLA ${ }^{2131}$, respectively
} 


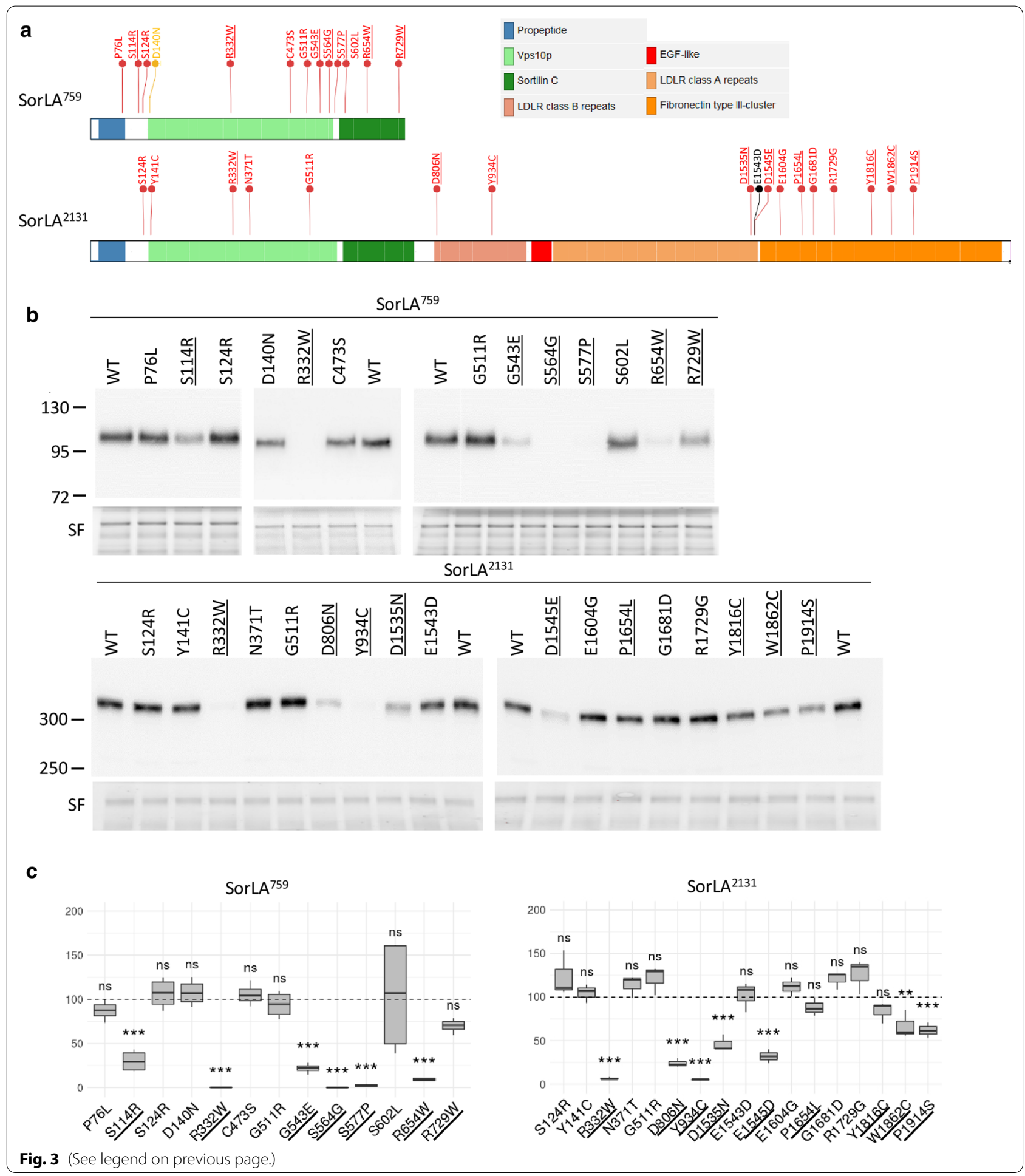

plasma membrane (Fig. 5c, Additional file 1: Fig. S4), suggesting that SorLA maturation is a prerequisite to its proper transport to the plasma membrane. Quantification of total plasma membrane-associated SorLA protein showed that the S124R and N371T variants presented a level of plasma membrane-associated SorLA similar to the wild-type protein (Fig. 5, c, d, Additional file 6: Table S4). In contrast, 2 of the 3 maturation-defective variants (R332W and R654W) showed statistically significant lower levels of mature SorLA protein at the 


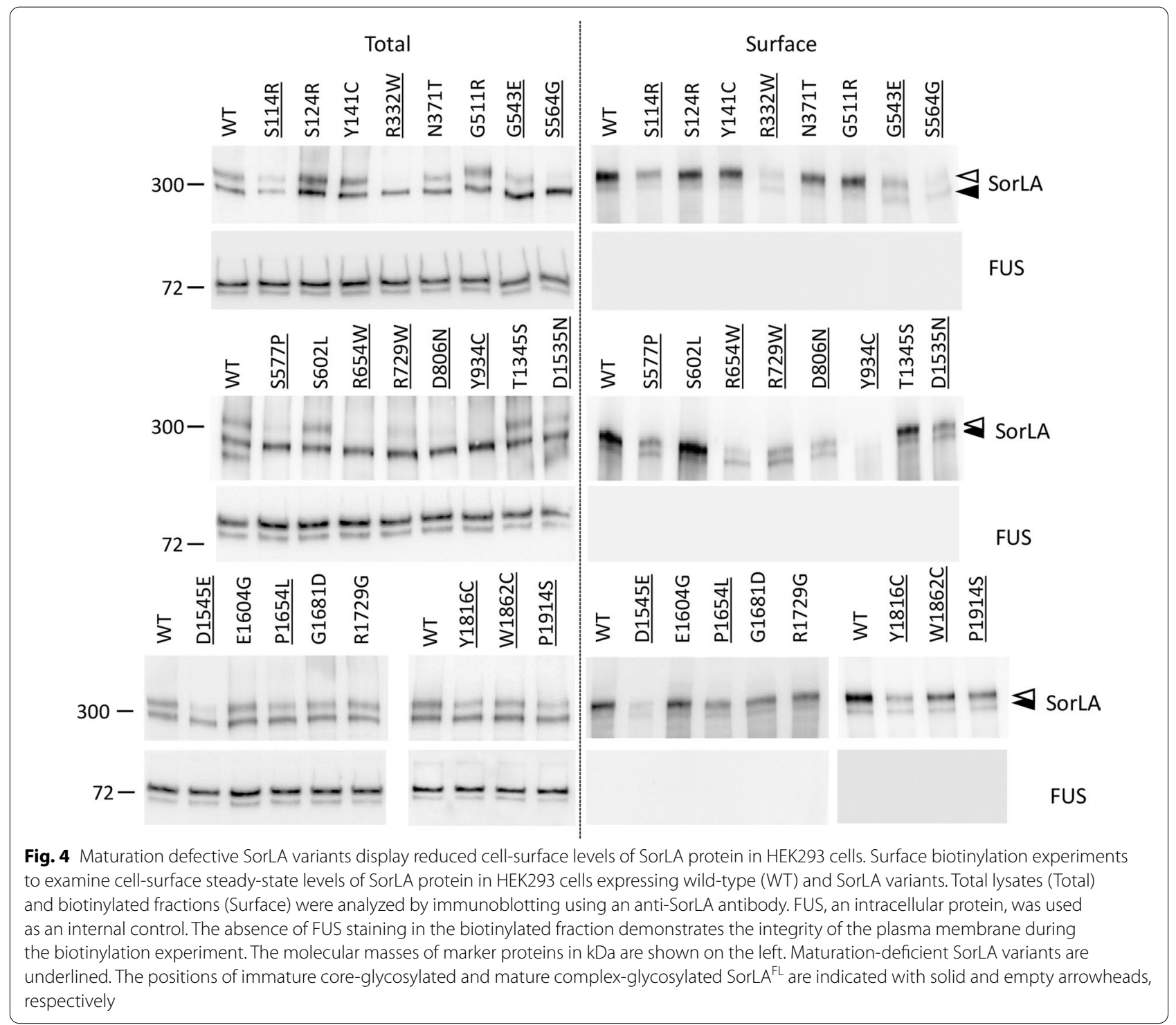

plasma membrane. The presence of the S577P variant also resulted in lower levels of SorLA at the plasma membrane, but levels were not statistically different from the wild-type form after correction for multiple testing. The amount of SorLA protein at the plasma membrane correlated with the amount of the mature form, the R654W showing the strongest effect with almost no detectable protein at the plasma membrane. The cytosolic FUS protein was used as control to exclude nonspecific labelling of the intracellular fractions. As expected, no FUS staining was detected in the biotinylated fraction.

Altogether, these data obtained in hiPSC lines expressing the R332W, S577P and R654W missense SORL1 variants at endogenous levels confirmed that these SorLA variants exhibit diverse degrees of maturation defects and lower levels of mature protein at the plasma membrane.

\section{Retention of the SorLA maturation defective variants in endoplasmic reticulum}

As SorLA maturation occurs during its transport from the endoplasmic reticulum towards the plasma membrane through the Golgi apparatus, we hypothesized that maturation-defective SorLA variants should present a disturbed subcellular localization with a redistribution within organelles involved in the secretory pathway. To address this issue, we performed immunofluorescence experiments and colocalization analyses on wild-type or CRISPR/Cas9-edited hiPSCs. 


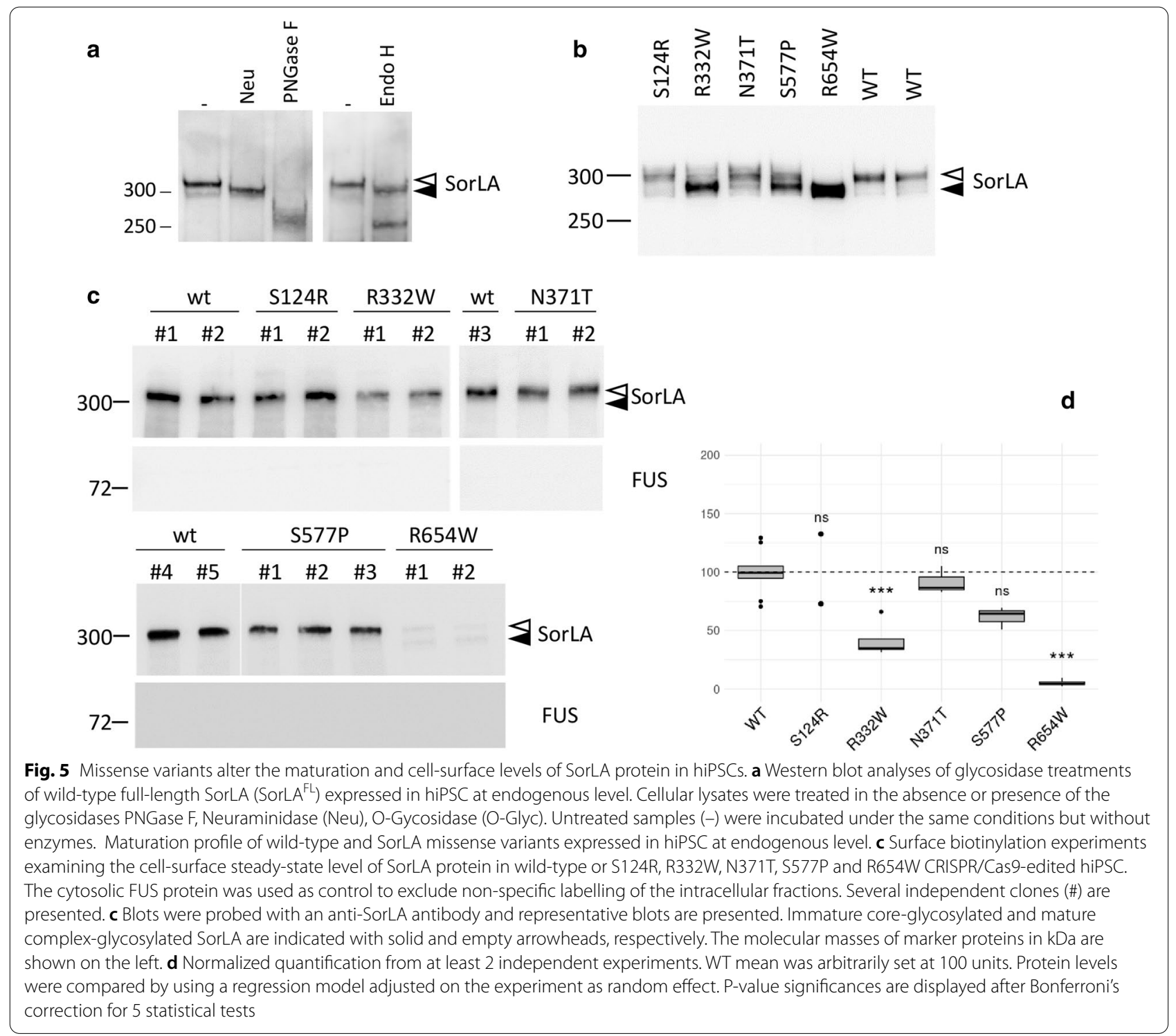

In accordance with previous reports [11, 41, 42], SorLA immunostaining in wild-type hiPSCs revealed a polarized localization, both in large perinuclear vesicles, and in smaller vesicles dispersed throughout the cytoplasm (Fig. 6). In the S124R and N371T hiPSCs, the subcellular distribution of SorLA protein was similar to that observed in wild-type cells. In contrast, the R332W and R654W hiPSCs presented only small SorLA-positive vesicles with widespread distribution throughout the cytoplasm. The S577P hiPSCs showed an intermediate distribution of SorLA, with fewer large vesicles close to the nucleus and larger amounts of small vesicles dispersed in the cytoplasm compared to the wild-type cells. SORL1 knockout (KO) hiPSCs were used as a negative control (Fig. 6, Additional file 1: Fig. S5).
Next, we performed immunofluorescence co-labelling of SorLA with Cyclophilin B and Rab5 as markers of the endoplasmic reticulum and the early endosomes, respectively (Additional file 1: Fig. S6, S7). The extent of SorLA colocalization with the two markers was assessed by calculating the M1 Manders' coefficient [37] (Fig. 7, Additional file 1: Fig. S8). Regarding the overlap of SorLA with the Cyclophilin B, the M1 coefficients did not statistically differ between the wild-type, S124R and N371T hiPSCs, indicating similar proportions of SorLA in the endoplasmic reticulum. In contrast, we observed statistically higher M1 coefficients for the R332W, R654W and S577P hiPSCs, indicating a higher proportion of SorLA proteins in the endoplasmic reticulum. Note that the variation of the M1 coefficient was less pronounced for the S577P 


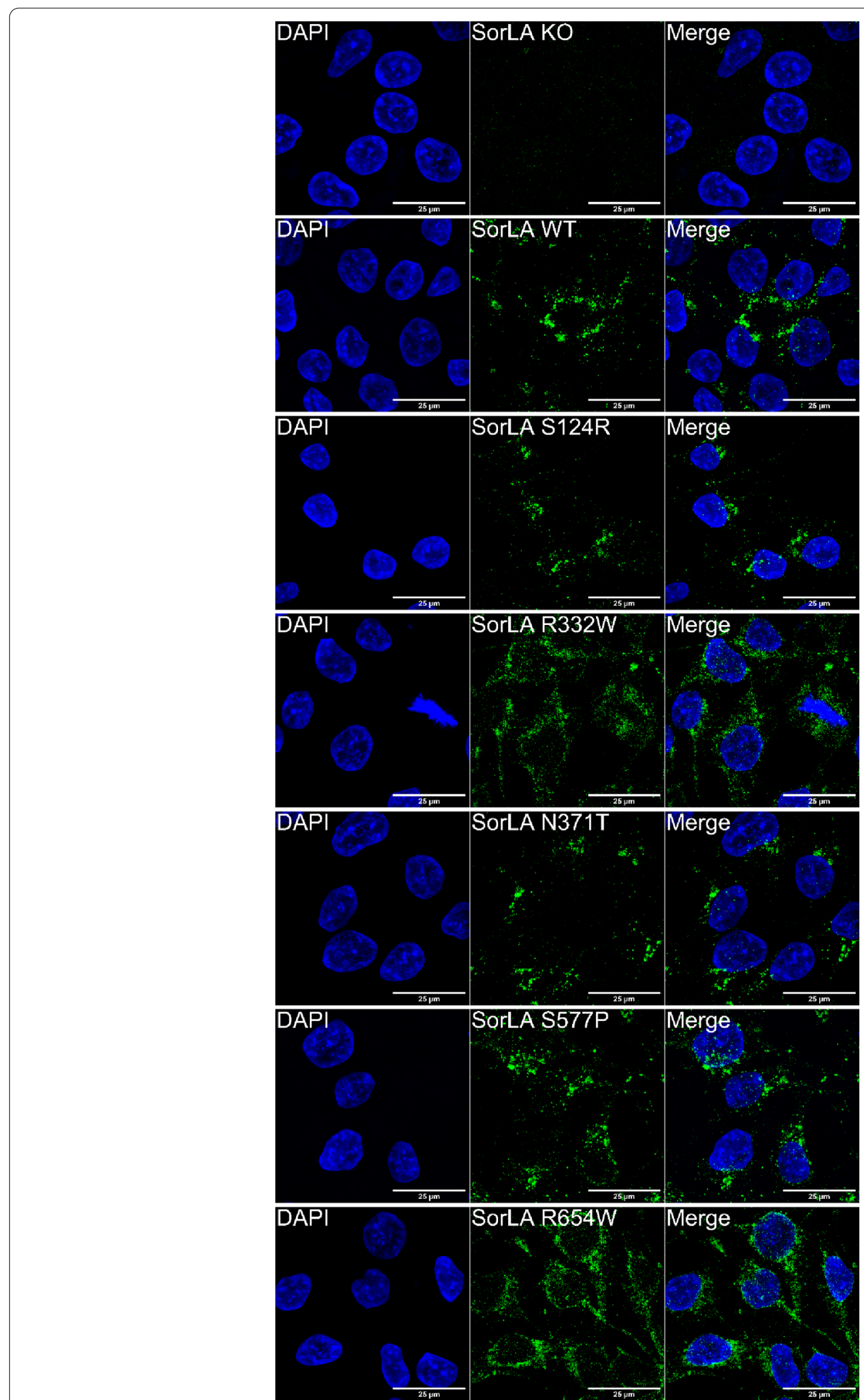

Fig. 6 Subcellular localization of the SorLA protein in wild-type and CRISPR/Cas9-edited human iPSC lines. In green, SorLA immunostaining. In blue, DAPI counter-staining 

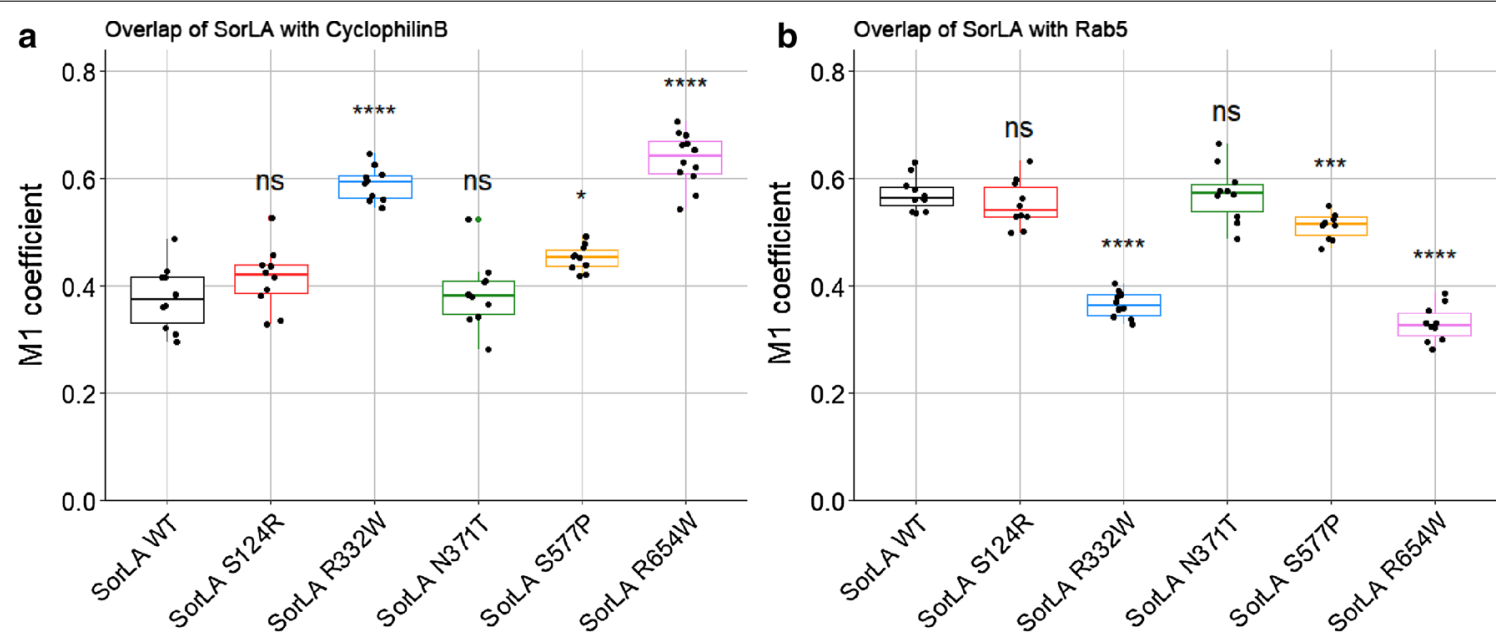

Fig. 7 Colocalization analyses of SorLA with markers of the endoplasmic reticulum and early endosomes in wild-type and CRISPR/Cas9-edited human iPSC lines. Overlap of SorLA staining with cyclophilin B (a) and Rab5 (b). Comparaison of M1 Manders' coefficients between SorLA genotypes was performed by the Welch's t-test, and p-value significances after Bonferroni's correction are represented above the box plots. For colocalisation analyses, more than ten images were acquired for each co-labelling

variant (Fig. 7a, Additional file 6: Table S4). Regarding the overlap of SorLA with Rab5, we observed inverse variations of the M1 coefficients. Accordingly, the M1 coefficients did not statistically differ between the wild-type, S124R and N371T hiPSCs, whereas the M1 coefficients were statistically smaller in the R332W, R654W and S577P hiPSCs compared to the wild-type cells, indicating a lower proportion of SorLA in early endosomes. Again, the variation of the M1 coefficient was less pronounced for the S577P variant (Fig. 7b, Additional file 6: Table S4).

Altogether, these data indicate that the subcellular distribution of the S124R and N371T SorLA variants is similar to the wild-type form of the protein, in accordance with a wild-type trafficking pattern. In contrast, the three maturation-defective variants (R332W, S577P and R654W) show diverse degrees of subcellular mislocalization, with a smaller proportion of SorLA in early endosomes and a higher proportion of SorLA in the endoplasmic reticulum, consistent with trafficking defects leading to their partial retention in this compartment.

SorLA variants associated with impaired cellular trafficking result in increased $A \beta$ secretion in hiPSCs

We next tested the impact of the S124R, R332W, N371T, S577P, R654W SORL1 variants on A $\beta$ secretion. The levels of $A \beta 40$ peptides secreted in the culture media from wild-type, S124R, R332W, N371T, S577P, R654W or KO

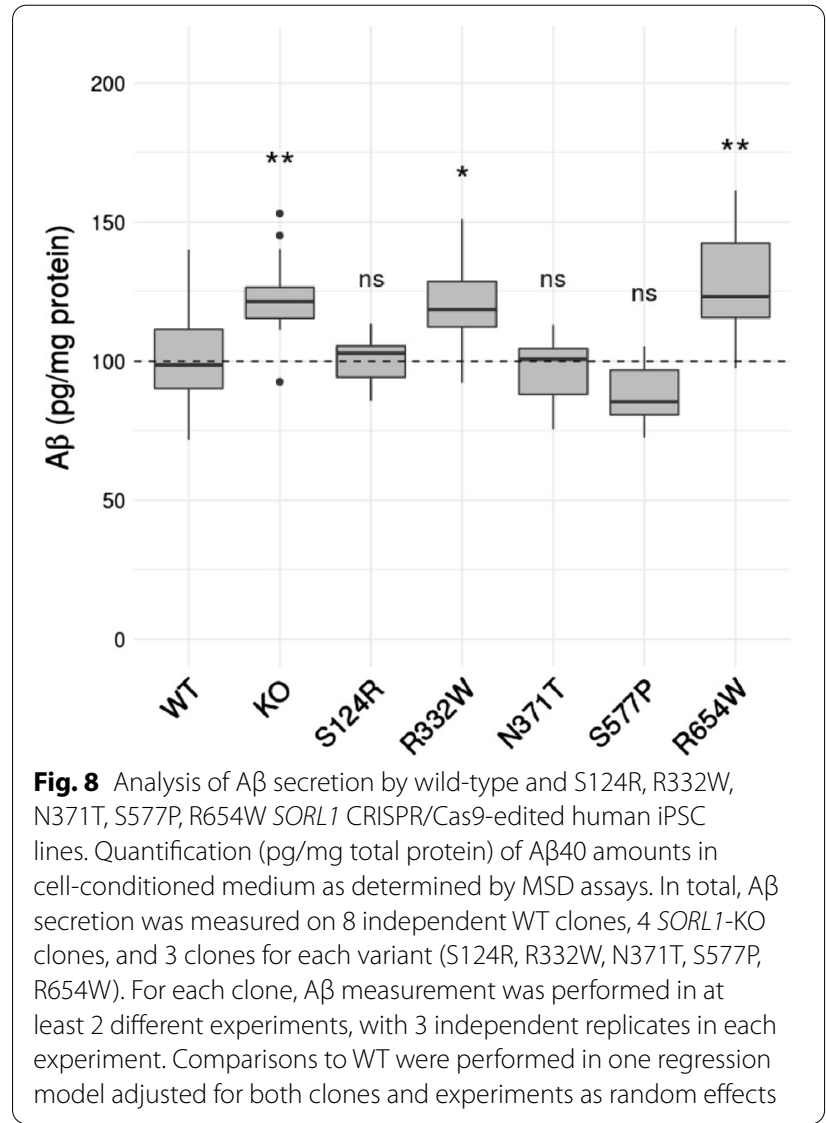




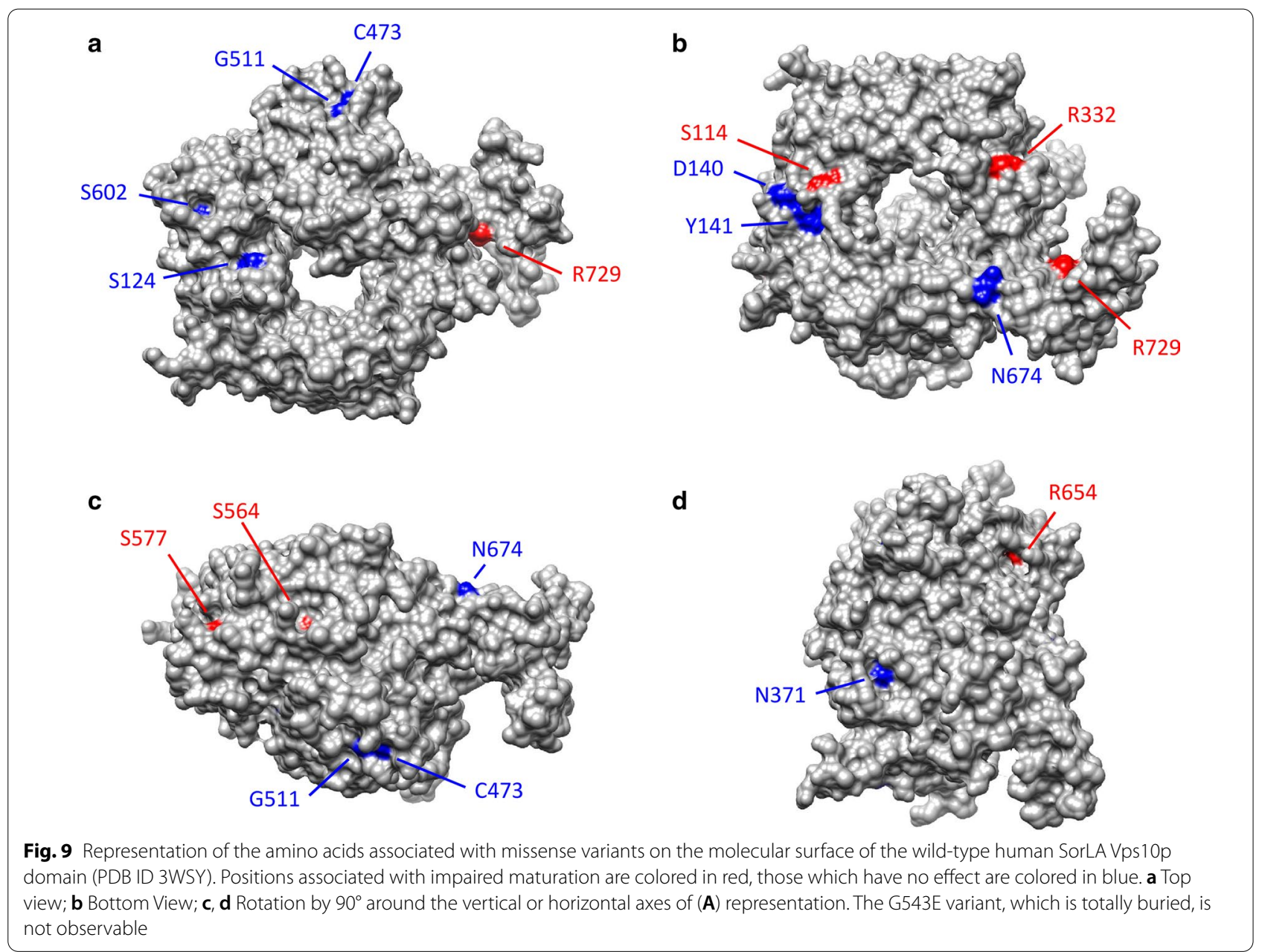

SorLA hiPSCs were measured using the MSD technology (Fig. 8, Additional file 6: Table S4). As expected, $\mathrm{KO}$ SorLA hiPSCs resulted in a significant increase in A $\beta 40$ secretion $(123.2 \pm 14.1 \%$ of control value, $p<0.001)$. The S124R, N371T and S577P mutants did not show increased $A \beta 40$ secretion compared to wild-type hiPSC (100.4 $\pm 8.9,99.1 \pm 11.0$, and $94.7 \pm 12.6$, respectively). In contrast, the R332W and R654W mutants, which showed the most obvious effect on SorLA maturation, led to significantly higher $\mathrm{A} \beta 40$ secretion $(121.4 \pm 14.4 p<0.01$ and $127.2 \pm 19.6 p<0.001$, respectively).
Impaired cellular trafficking of SorLA protein could be due to subtle variations of the protein 3D structure resulting from changes in the interatomic interactions

The DynaMut webserver [39] was used to evaluate the impact of missense variants on SorLA structural stability. We focused on variants localized in the Vps10p domain of SorLA whose crystal structure was available in the Protein Data Bank ([38], PDB ID 3WSY). Both variants corresponding to maturation-defective proteins (S114R, R332W, G543E, S564G, S577P, R654W and R729W) or displaying a similar maturation profile as the one 
observed for WT-SorLA (S124R, D140N, Y141C, N371T, C473S, G511R, S602L and N674S) exhibited similar profiles (Additional file 7: Table S5). Moreover, the variations in the Gibbs Free Energy values was relatively small, suggesting that the mutations had no major impact on SorLA global structure.

To gain further insight into the effect of missense variants on SorLA function, amino acids associated with variants were displayed on the protein structure (Fig. 9). The 8 variants presenting a wild-type maturation profile corresponded to exposed amino acids as expected for neutral variations [43]. Positions corresponding to impaired maturation variants were distributed over the Vps10p domain. The associated residues were either buried (G543E) or partially buried (S564G, S577P, R654W, Fig. 9c, d) or partially exposed (S114R, R332W, R729W, Fig. 9a, b) but did not correspond to residues maintaining the integrity of Vps10p ten-bladed $\beta$-propeller or 10CC domains. These observations were consistent with the lack of major effects on the protein structure stability predicted by DynaMut and indicated that the altered function was rather linked to modifications of macromolecular interactions such as hydrophobic and electrostatic interactions, van der Waals forces and hydrogen bonds.

The interatomic interactions of the 3 impaired maturation and trafficking-deficit variants (R332W, S577P, and R654W) and of the 2 maturation-competent variants (S124R and N371T) studied in hiPSC were further studied in detail (Fig. 10). Consistent with its neutral nature, the mutation of a Serine to an Arginine in the S124R variant, despite the size difference between the two residues, led to very few changes in the intermolecular interactions (Fig. 10A and $\mathrm{A}^{\prime}$ ). Similarly, for the N371T variant, the interaction network, except a hydrogen bond between N371 and E388, was conserved (Fig. 10C, $C^{\prime}$ ). On the contrary, for the last three variants (R332W, S577P, and R654W), the interaction network as well as the contact nature were modified. In the $\mathrm{R} 332 \mathrm{~W}$ variant, the substitution of a long polar by a bulky aromatic sidechain gave rise to additional contacts, such as cation- $\pi$ interactions with R279 and to the loss of the hydrogen bond with D304 (Fig. 10B and $\mathrm{B}^{\prime}$ ). Other existing interactions were altered, going from polar or ionic to hydrophobic. In a similar manner, for S577P, the replacement of a polar by a hydrophobic sidechain, changed the nature of the interaction network, polar contacts being switched for hydrophobic ones (Fig. 10D, D'). As for R332W, in the R654W variant, the substitution of an Arginine by a Tryptophane led to the appearance of cation- $\pi$ interactions with G547 and of hydrophobic contacts with E567, as well as to the disappearance of the H-bond with G547 (Fig. 10E, E').
Altogether, these analyses suggest that altered maturation and trafficking of SorLA protein could be due to subtle variations of the protein structure resulting from changes in the interatomic interactions.

\section{Discussion}

Previous functional studies proposed two distinct pathological molecular mechanisms for SORL1 missense variants: (i) reduced binding affinities for APP and thus increased amyloidogenic processing of APP, by favouring its targeting to the late endosome compartments where APP is cleaved into $A \beta$, or (ii) reduced $A \beta$ binding that may affect the ability of SorLA to direct $A \beta$ peptides to lysosomes for degradation [21, 28, 29]. In the present study, we identified a novel pathological molecular mechanism, in which rare missense SorLA variants show protein maturation and trafficking defects.

In this study, we analyzed 70 rare missense SORL1 variants. When overexpressed in HEK293 cells, 15 of these 70 variants (S114R, R332W, G543E, S564G, S577P, R654W, R729W, D806N, Y934C, D1535N, D1545E, P1654L, Y1816C, W1862C, P1914S) showed maturation and plasma membrane targeting-deficient phenotype. Five of these variants (S124R, R332W, N371T, S577P, and $\mathrm{R} 654 \mathrm{~W}$ ) were further studied in details in CRISPR/Cas9modified hiPSCs in order to avoid any bias related to the overexpression of the SorLA protein. When expressed at endogenous levels, the maturation defective profile of the R332W, S577P, and R654W SorLA variants was confirmed. We further demonstrated that these variants were largely retained in the endoplasmic reticulum, explaining the higher content of high mannose type $\mathrm{N}$-glycans observed. This resulted in a reduction in the delivery of SorLA mature protein to the plasma membrane and to the endosomal system. Importantly, this maturation/trafficking-defective process was associated with a clear increase of $A \beta$ secretion for the R332W and R654W variants, demonstrating a loss-of-function effect of these SorLA variants regarding this ultimate readout, and a direct link with AD pathophysiology. Furthermore, structural analysis of the impact of missense variants on SorLA protein revealed that impaired cellular trafficking of SorLA could be due to subtle variations of the protein structure resulting from changes in the interatomic interactions.

Defects in protein trafficking and ER retention have been shown to be a common cellular mechanism involved in the pathogenesis of a wide range of inherited human diseases, such as cystic fibrosis [44] or kidney diseases [45]. Mutations act by impacting protein folding and conformational stability, thus interfering with 

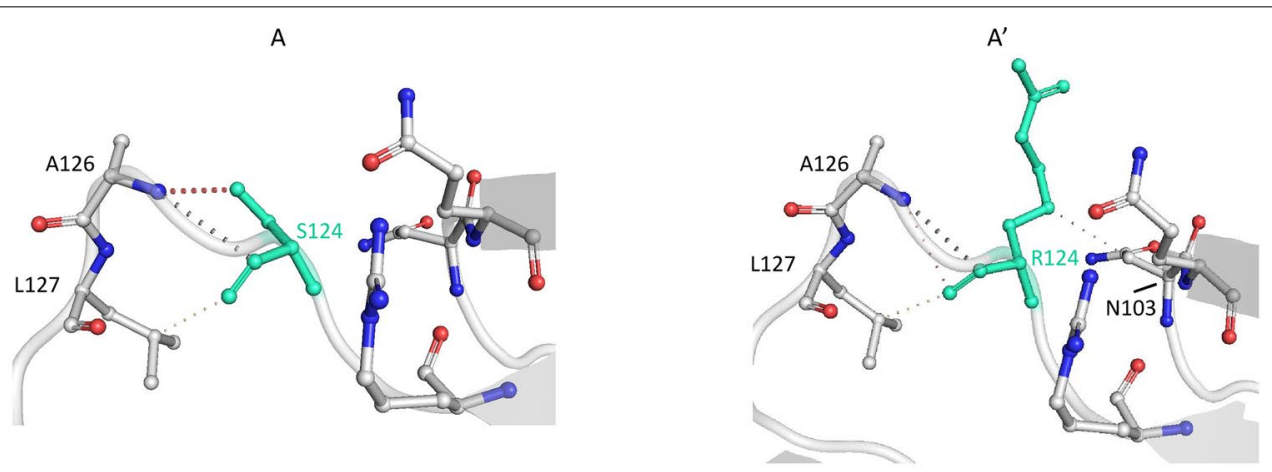

S124R
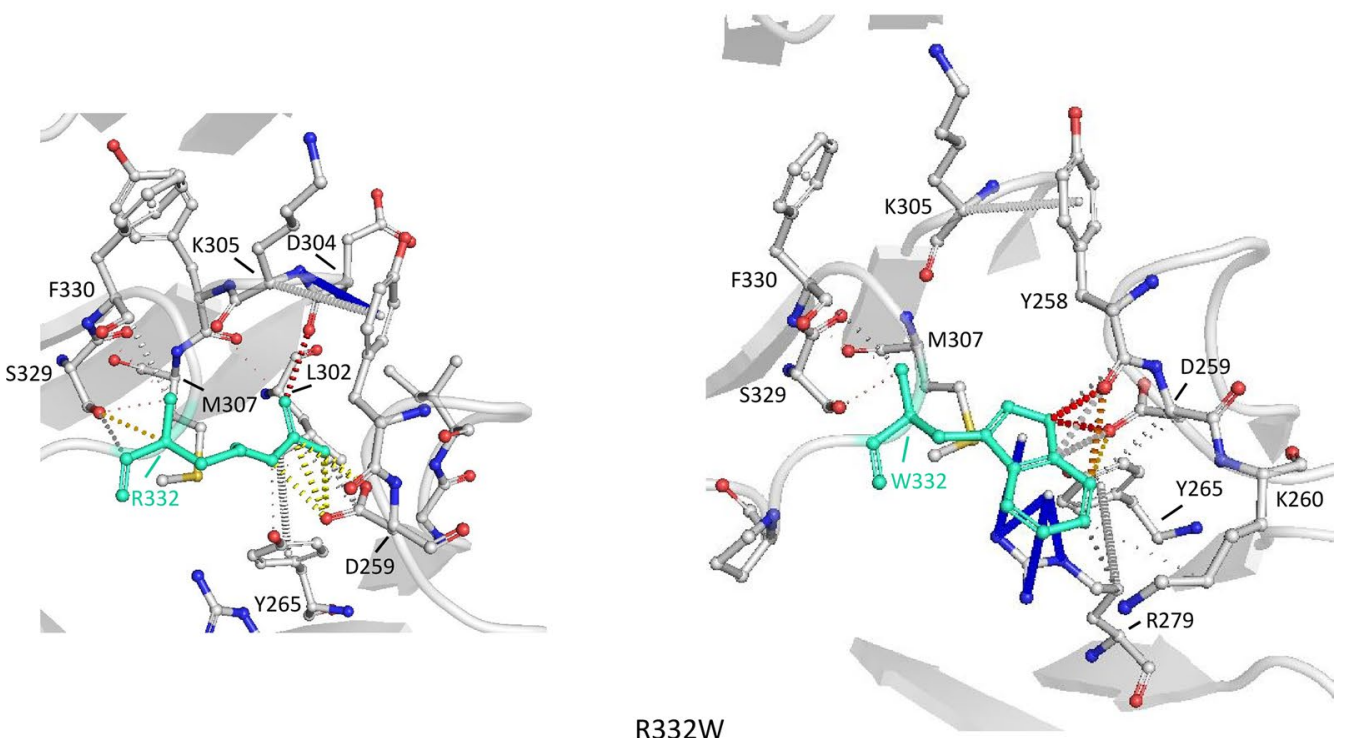

C

R332W
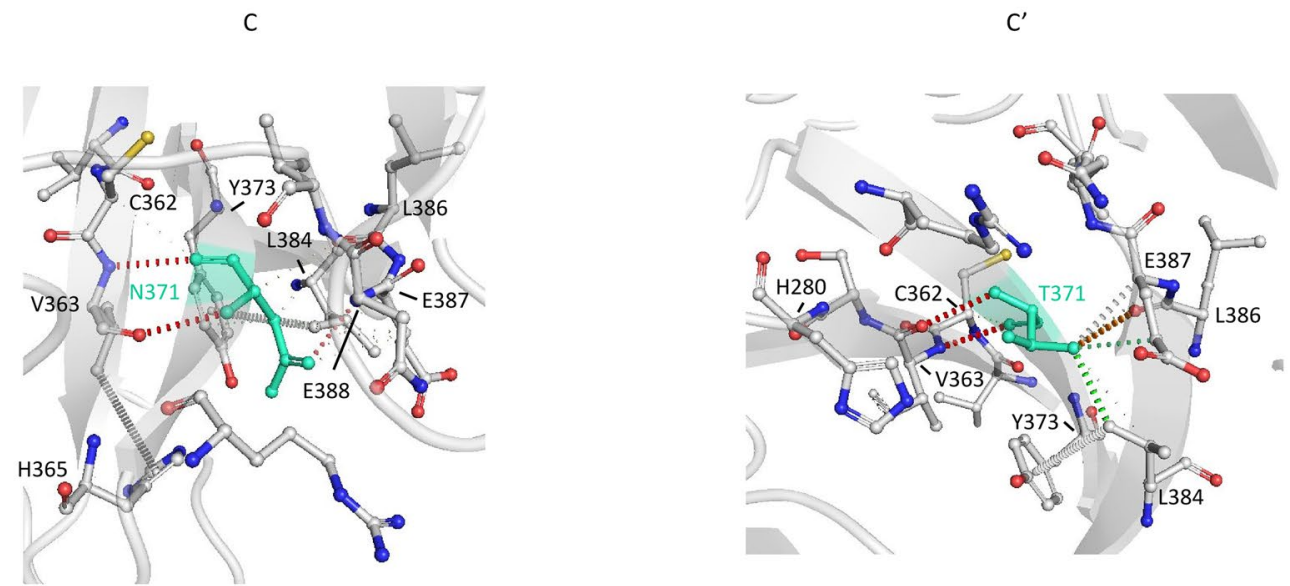

N371T

Fig. 10 Prediction of interactions between amino acid residues in human SorLA Vps10p domain (PDB ID 3WSY) by DynaMut. The WT and variant residues are colored in green and are represented as sticks alongside the surrounding residues that are involved in any type of interactions. Amino acids involved in interactions are labelled in black and labels are placed next to Ca atoms. The colors of the contacts are based on the following: red, polar (including hydrogen bonds); orange, weak polar; yellow, mixed ionic van der waals; blue, cation $\pi$; and gray, hydrophobic (including carbon $\pi$ ). Interactions between the local amino acids of the WT and ( $\mathbf{A}$ and $\mathbf{A}^{\prime}$ ) the S124R variant; (B and $\mathbf{B}^{\prime}$ ) the R332W variant; the N371T variant (C and $\mathbf{C}^{\prime}$ ); the S577P variant ( $\mathbf{D}$ and $\mathbf{D}^{\prime}$ ); the R654W variant ( $\mathbf{E}$ and $\mathbf{E}^{\prime}$ ) 


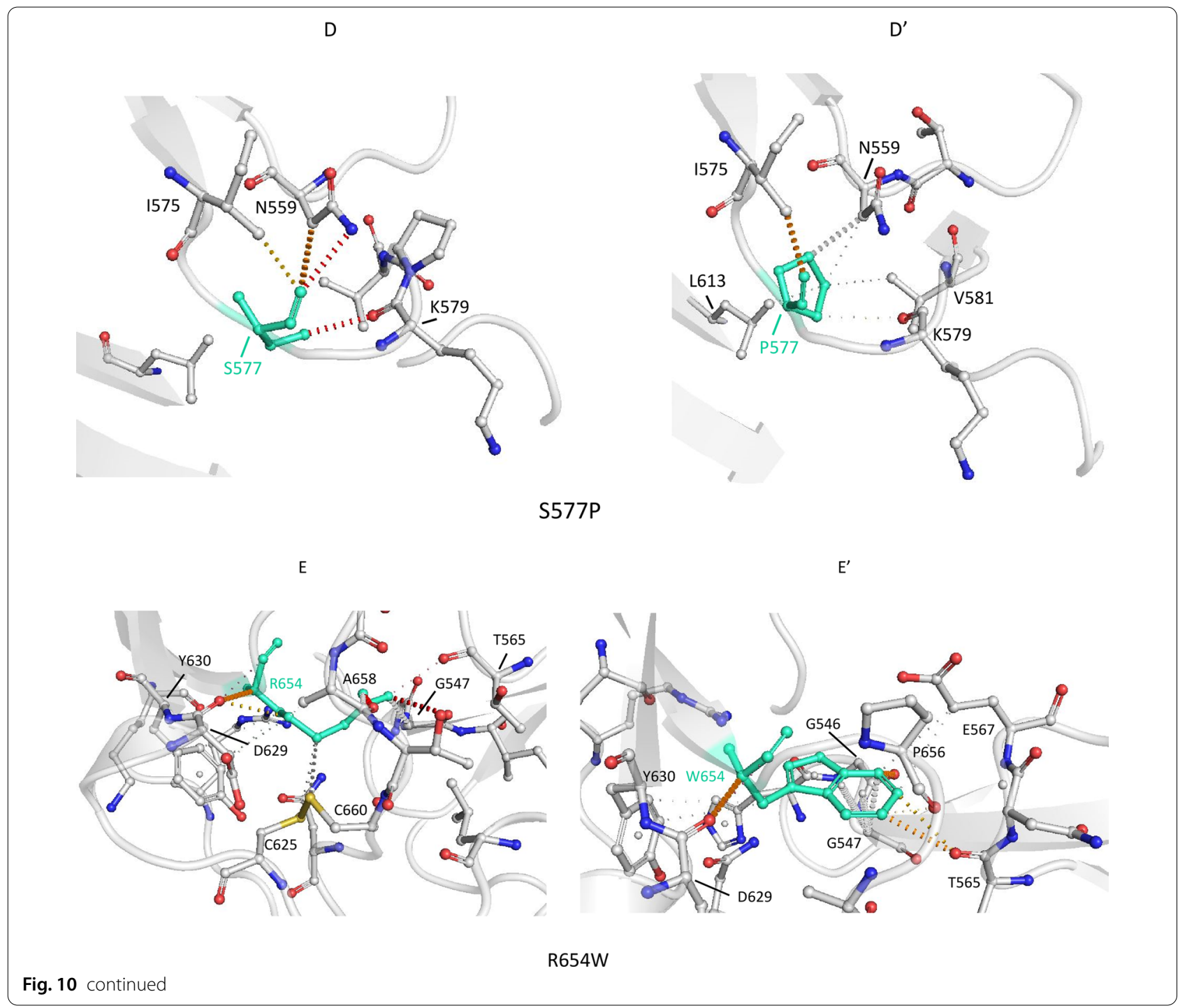

efficient post-translational modification and subsequent protein trafficking. The transport of misfolded glycoproteins in the ER is inhibited by a process called quality control [46]. Pending the severity of the conformational defect, partially folded proteins can be chaperoned to attain their native conformation whereas irreversibly misfolded polypeptides are disposed via the ER-associated degradation pathway (ERAD).

Our strategy, based on an initial screening in HEK293 cells followed by a subsequent validation in CRIPSR/ Cas9-modified hiPSCs, revealed a few discrepancies between our cellular models. The effect of the R332W and S577P variants on SorLA maturation profile, which was similar to that of the R654W variant in HEK293 cells, appeared to be weaker in hiPSCs. Indeed, some mature forms of R332W and S577P SorLA proteins were produced and addressed to the plasma membrane in hiPSCs.
Overexpression may therefore exacerbate the effect of some variants. HEK293 cells overexpressing SorLA would contain such large amounts of the protein that the machinery responsible for further post-translational processing and the ER quality-control system may well be overloaded, leading to a disproportionate accumulation of immature forms. The HEK293 cells might thus be a sensitive model to reveal variants with low or moderate impact on SorLA maturation.

Cell-surface biotinylation experiments in CRISPR/ Cas9-modified hiPSCs showed that, when expressed at endogenous levels in hiPSCs, only the glycosylated mature form of the SorLA protein was detected at the plasma membrane, suggesting a prerequisite of SorLA maturation for its proper transport to the plasma membrane. Interestingly, although at very low level, the 
R654W variant exists at the cell membrane in two forms: an immature and a mature form. ER-retained proteins are typically degraded in a pre-Golgi compartement. However, in some diseases, it has been shown that ERretained mutant proteins are not effectively degraded and might accumulate, which seems to be also the case for the immature R654W mutant protein. Thus, when present in a great amount in the ER, some immature species and/ or misfolded protein might bypass the ER quality control mechanism and be transported to the plasma membrane. These data are fully consistent with our results obtained in HEK293 cells in which we detect a disproportionate accumulation of immature forms, and the presence of both immature and mature forms at the plasma membrane. The presence of both species at the plasma membrane in HEK293 cells, which is consistent with the data previously reported by other groups [3, 9], could therefore be related to the overexpression of SorLA and the saturation of the ER quality control system.

The 15 SorLA variants that showed deficiency in protein maturation and plasma membrane targeting in this study (S114R, R332W, G543E, S564G, S577P, R654W, R729W, D806N, Y934C, D1535N, D1545E, P1654L, Y1816C, W1862C, P1914S) are distributed along the entire extracellular part of the protein, with the exception of the EGF-like and the LDLR class A repeats domains, suggesting that these two domains are less sensitive to amino acid substitutions regarding this particular phenotype. We wondered how these 15 amino acid substitutions could impact SorLA protein global structure, stability and/or flexibility. None involves an Asparagine (N) potentially involved in N-glycosylation anchoring. Nor do they create ER-resident motifs, such as KDEL and dilysine. The Y1816C and W1862C variants that lead to the introduction of additional Cysteine residues within the Fibronectin type III-cluster may be involved in the formation of incorrect intramolecular disulfide bonds. The analysis of the impact of missense variants on SorLA structural stability, focusing in the Vps10p domain of SorLA whose crystal structure was available [38], suggested that the mutations had no major impact on SorLA global structure. However, the detailed study of interatomic interactions of the 3 impaired maturation variants (R332W, S577P, and R654W) showed that the interaction network as well as the contact nature were modified. Alteration of the interatomic interactions could result in subtle variations of the protein $3 \mathrm{D}$ structure and folding stability.

The study in hiPSCs allowed us to highlight a gradual effect of the variants, with S577P $<$ R332W $<$ R654W. This gradual effect could be observed on all the phenotypes assessed: maturation defects of the protein, retention in the ER, release to the plasma membrane, endosomal localization and $A \beta$ production. This could be due to different severities of the folding defect present in the mutated protein and/or the ability of the ER quality control system to correct these. We can assume that the impact of the R332W and S577P variants on protein conformation in this cellular model are more moderate, and that the folding process is able to occur, at least partially.

In contrast to the R654W variant that was barely detected as mature species, significant amounts of mature forms of R332W and S577P SorLA mutant proteins were produced in hiPSCs, raising the question of their functionality regarding APP metabolism. The expression of the R332W variant resulted in a significant increase of $A \beta$ secretion. This effect on extracellular $A \beta$ levels may result from an insufficient amount of mature SorLA protein in the appropriate cellular compartments to ensure its function with respect to APP metabolism, and/or a direct impact of this variant on SorLA function on APP metabolism, both not being exclusive. Regarding the S577P variant, we observed no effect on extracellular $A \beta$ levels. This indicates that, in our cellular model, the amount of mature protein produced is sufficient and that this mutant protein is functional regarding APP metabolism. The absence of effect of the S577P variant on A $\beta$ secretion in hiPSCs does not preclude its role in the AD neurodegenerative process. Indeed, age-associated deterioration of cellular machinery leads to an increase in the occurrence of protein misfolding, accumulation and aggregation, due in part to the gradual decay of ER chaperoning systems. It is therefore possible that this S577P variant, identified in AD patients, has a long-term effect that would not be featured by our shortterm culture experiments. As we cannot exclude specific effects of SorLA variants during aging conditions, neither can we exclude a cell-specific effect. It would be interesting to model this variant in a neuronal environment with longlasting experiments to determine whether it is involved in $\mathrm{AD}$ or if it should be considered as rare sequence variants with no functional significance and no relevance with $\mathrm{AD}$. Regarding the variants identified in AD patients that did not display drastic maturation and transport defects in our cellular model, our data do not preclude any role for these variants in the $\mathrm{AD}$ neurodegenerative process. Putative other mechanisms leading to a SorLA loss of function should be assessed, such as APP and A $\beta$ binding.

In this study, we analysed iPSC lines carrying mutations at the homozygous state in order to clearly distinguish the maturation profile of mutant SorLA from the wild-type form of the protein. In patients, SORL1 missense variants are found at the heterozygous state, and we can expect that in the early years, the quantity and quality of SorLA protein encoded by the wild-type allele, and potentially by the mutated allele, is sufficient to ensure protein function. However, as individuals age, the decline in cellular quality control systems will lead to 
the production of more and more non-functional proteins, resulting in a progressive loss of SorLA function.

Interestingly, genomic data appear to be in line with our in vitro experiments, since we observed an apparent inverse correlation between the frequency of the variants and the measured effect in our experiments. Indeed, the frequency in large control databases such as gnomAD v2.1.1, non-neuro subset, ranged from a full absence (R654W), to the presence in 4 individuals (R332W), 9 indididuals (S577P) and 304 individuals (N371T), out of 114,679 controls. This is consistent with the inverse correlation between odds ratios and variant frequency found in case-control studies [24, 27]. Conversely, the S124R variant, which is recurrent among $\mathrm{AD}$ cases (2 French cases, through two distinct genomic changes), is absent in controls and in the gnomAD database. Thus, the existence of two distinct genomic changes leading to a same missense change in two unrelated patients suggests that this amino acid position should be further studied in other models, as for the S577P, and despite the absence of modification of $A \beta$ secretion in hiPSCs after $24 \mathrm{~h}$ secretion.

Of note, case-control studies assessing the putative effect of SORL1 missense variants mainly relied on the use of in silico predictions (e.g., the Mis1-2-3 classification). Interestingly, all 15 variants showing an impact on SorLA maturation in HEK293 cells belonged to the Mis3 category. However, such in silico predictions are not a sufficient argument for using SORL1 variants in a clinical context, as not all Mis3 variants might eventually behave as loss-of-function variants, first, and because there may be some diversity in the types and strengths of effects among missense variants, second. Overall, we conservatively consider that at least part of the variants studied here can now be rated as definite AD risk factors, namely R332W and R654W, given their deleterious effect on SorLA maturation confirmed in both cellular models and associated with clearly increased secretion levels of $A \beta$ peptides. Further work remains necessary to confirm a definite role for the other $13 / 15$ variants in a model without overexpression and to assess the degree of associated loss of SorLA function.

\section{Conclusion}

In conclusion, by assessing missense variants located throughout the coding sequence of the SORL1 gene among rare variants found in Alzheimer disease patients, we identified a novel mechanism leading to a loss of SorLA function. A subset of the variants induced a likely misfolded protein, thus altering the protein maturation and trafficking, and eventually leading to a loss of the protective function of SorLA towards $A \beta$ secretion.
SORL1 maturation defective variants are a novel mechanism directly link to AD pathophysiological mechanisms.

\section{Abbreviations}

AD: Alzheimer's disease; EOAD: Early-onset Alzheimer's disease; ER: Endoplasmic reticulum; hiPSCs: Human induced pluripotent stem cells; KO: Knockout; NMD: Nonsense-mediated decay; PTV: Protein-truncating variants; SORL 1: Sortilin-related receptor 1; sSorLA: Soluble SorLA; TGN: Trans-Golgi network.

\section{Supplementary Information}

The online version contains supplementary material available at https://doi. org/10.1186/s40478-021-01294-4.

Additional file 1:: Supplementary figures.

Additional file 2.: Legends of the supplementary figures.

Additional file 3: Table S1: Summary of analyses performed in HEK293 cells expressing wild-type (WT) or missense variants of SorLA, or in CRISPR/Cas9-edited hiPSC. Nucleotide positions are relative to canonical transcript NM_003105.5. The nucleotide change on CDNA, the corresponding amino acid change on the protein, and the location along the protein (Functional domain) are indicated. The Mis3, Mis2, and Mis0-1 missense variants examined in this study are shown in red, orange and black, respectively. For each variant, the number of carriers among our cohort (or among cohorts described in the litterature when applicable) and the corresponding frequency are indicated, as well as the allele count and the corresponding frequency in the gnomAD v2.1 database (only including individuals of European ancestry, and classified as "non-neuro"). Regarding the functional studies inHEK293 cells and iPSCs, a (Y) indicates an impact of the variants on SorLA protein maturation, transport or function and a $(\mathrm{N})$ indicates no change from the wild-type form of the protein.

Additional file 4: Table S2: Primer pairs used to introduce SorLA genetic variations into the wild-type sequence by site-directed mutagenesis

Additional file 5: Table S3: Sequences of guide RNAs, ssODNs and primer pairs used for CRISPR/Cas9 gene editing and clone screening. For each variant, the nucleotide change (as a function of the genomic position and SORL1 CDNA) and the resulting protein change are indicated, together with the sequences of the guide RNAs, the corresponding ssODNs and the Sanger sequencing primer pairs. On ssODN sequences, lowercases correspond to intronic sequences. The position of the variant is indicated in red and the corresponding nucleotide triplet is underlined. The synonymous variants introduced to preclude re-cutting by the Cas 9 are indicated in green and the corresponding nucleotide triplet is underlined.

Additional file 6: Table S4: p-Values of statistical analyses

Additional file 7: Table S5: Predicted results obtained with the Dynamut Server upon mutation. Mutations corresponding to maturation-defective proteins are colored in red. No correlation between impaired maturation and missense variants was observed. Indeed S114R, R332W and R654W were predicted to result in more stable structures (positive $\Delta \Delta \mathrm{G}$ ) while G543E, S654G, S577P and R729W were associated with a decrease of stability (negative $\triangle \Delta G$ ).

\section{Acknowledgements}

We thank Pr Muriel Bardor, Laboratoire Glyco-MEV EA 4358, University of Rouen Normandie, Pr Maité Montero, DC2N, University of Rouen Normandie, and Damien Shapman, PRIMACEN University of Rouen Normandie, for helpful discussion. We thank Muriel Bardor for critical reading of the manuscript. Light microscopy was performed at the PRIMACEN imaging platform (Rouen University, France). We thank Cyril Pottier and Kilan Le Guennec for their help on plasmid synthesis. Molecular graphics and analyses were performed with UCSF Chimera, developed by the Resource for Biocomputing, Visualization, and Informatics at the University of California, San Francisco, with support from NIH P41-GM103311. 


\section{Authors' contributions}

Conceptualization: A.R-L., L.M., S.F., M.L., G.N.; Formal analysis: M.L, L.M, A.R-L, C.S., S.F., I.S-M, L.G., O.Q; Funding acquisition: A.R-L., M.L., G.N., D.C., T.F., I.S-M, L.G.; Investigation: A.R-L., L.M., S.F., C.S., S.P., G.R., S.R., L.G., I.S-M., M.L.; Methodology: A.R-L., L.M., S.F., C.S., G.R., L.G., I.S-M., M.L.; Supervision: M.L., G.N.; Validation: C.S., A.R-L., L.M., S.F., S.R., M.L.; Visualization: A.R-L., L.M., S.F., C.S., S.R., L.G., I.S-M., M.L., O.Q; Writing - original draft: A.R-L., L.M., S.F., C.S., L.G., I.S-M., M.L., G.N., D.C. All authors read and approved the final manuscript.

\section{Funding}

This work was co-supported by a grant from France Alzheimer association (AAPSM2019 - grant $n^{\circ} 1957$ ), the Fondation pour la Recherche Médicale (FRM, grant \# DEQ20170336711 and \# ARF201909009263 to C.S.), the Fondation Alzheimer (ECASCAD study) and the European Union and the Région Normandy (RIN-NICE). Europe gets involved in Normandy through the European Regional Development Fund (ERDF). This work was co-supported by the CNRMAJ (national reference center for young Alzheimer patients, Rouen, France). Contribution of I.S.M and L.G. was financially supported by University of Rouen Normandy, INSA Rouen Normandy, the Centre National de la Recherche Scientifique (CNRS), European Regional Development Fund (ERDF), Labex SynOrg (ANR-11-LABX-0029), Carnot Institute I2C, the graduate school for research XL-Chem (ANR-18-EURE-0020 XL CHEM), and by Région Normandie.

\section{Availability of data and materials}

All data generated or analysed during this study are included in this published article [and its supplementary information files].

\section{Declarations}

Ethics approval and consent to participate

Not applicable.

\section{Consent for publication}

Not applicable.

\section{Competing interests}

The authors declare that they have no competing interests.

\section{Author details}

${ }^{1}$ Department of Genetics and CNR-MAJ, Normandie Univ, UNIROUEN, Inserm U1245, CHU Rouen, FHU G4 Génomique, 76000 Rouen, France. ${ }^{2}$ Laboratoire COBRA (UMR 6014 \& FR 3038), Normandie Université, UNIROUEN, INSA de Rouen, CNRS, Rouen, France. ${ }^{3}$ Inserm U1234, Flow Cytometry Core - IRIB, Rouen, France.

\section{Received: 10 November 2021 Accepted: 15 November 2021}

\section{Published online: 18 December 2021}

\section{References}

1. Nicolas G, Charbonnier C, Campion D (2016) From common to rare variants: the genetic component of alzheimer disease. Hum Hered 81:129-141

2. Bellenguez C, Charbonnier C, Grenier-Boley B, Quenez O, Le Guennec K, Nicolas $G$ et al (2017) Contribution to Alzheimer's disease risk of rare variants in TREM2, SORL1, and ABCA7 in 1779 cases and 1273 controls. Neurobiol Aging 59:220.e1-220.e9

3. Jacobsen L, Madsen P, Jacobsen C, Nielsen MS, Gliemann J, Petersen CM (2001) Activation and functional characterization of the mosaic receptor SorLA/LR11. J Biol Chem 276:22788-22796

4. Fiete D, Mi Y, Oats EL, Beranek MC, Baenziger JU (2007) N-Linked oligosaccharides on the low density lipoprotein receptor homolog SorLA/LR11 are modified with terminal GalNAc-4-SO4 in kidney and brain*. J Biol Chem 282:1873-1881

5. Schmidt V, Sporbert A, Rohe M, Reimer T, Rehm A, Andersen OM et al (2007) SorLA/LR1 1 regulates processing of amyloid precursor protein via interaction with adaptors GGA and PACS-1. J Biol Chem 282:32956-32964

6. Fjorback AW, Seaman M, Gustafsen C, Mehmedbasic A, Gokool S, Wu C et al (2012) Retromer binds the FANSHY sorting motif in SorLA to regulate amyloid precursor protein sorting and processing. J Neurosci 32:1467-1480

7. Steentoft C, Vakhrushev SY, Joshi HJ, Kong Y, Vester-Christensen MB, Schjoldager KT-BG et al (2013) Precision mapping of the human O-GalNAC glycoproteome through SimpleCell technology. EMBO J 32:1478-1488

8. Hampe W, Riedel IB, Lintzel J, Bader CO, Franke I, Schaller HC (2000) Ectodomain shedding, translocation and synthesis of SorLA are stimulated by its ligand head activator. J Cell Sci 113(Pt 24):4475-4485

9. Christensen SK, Narimatsu Y, Simoes S, Goth CK, Vægter CB, Small SA et al. Endosomal trafficking is required for glycosylation and normal maturation of the Alzheimer's-associated protein sorLA. bioRxiv. 2020:2020.07.12.199885.

10. Hermey G, Sjøgaard SS, Petersen CM, Nykjaer A, Gliemann J (2006) Tumour necrosis factor alpha-converting enzyme mediates ectodomain shedding of Vps10p-domain receptor family members. Biochem J 395:285-293

11. Nielsen MS, Gustafsen C, Madsen P, Nyengaard JR, Hermey G, Bakke O et al (2007) Sorting by the cytoplasmic domain of the amyloid precursor protein binding receptor SorLA. Mol Cell Biol 27:6842-6851

12. Schmidt $V$, Subkhangulova A, Willnow TE (2017) Sorting receptor SORLA: cellular mechanisms and implications for disease. Cell Mol Life Sci 74:1475-1483

13. Huang TY, Zhao Y, Li X, Wang X, Tseng I-C, Thompson R et al (2016) SNX27 and SORLA interact to reduce amyloidogenic subcellular distribution and processing of amyloid precursor protein. J Neurosci 36:7996-8011

14. Dumanis SB, Burgert T, Caglayan S, Füchtbauer A, Füchtbauer E-M, Schmidt $V$ et al (2015) Distinct functions for anterograde and retrograde sorting of SORLA in amyloidogenic processes in the brain. J Neurosci Soc Neurosci 35:12703-12713

15. Andersen OM, Reiche J, Schmidt V, Gotthardt M, Spoelgen R, Behlke J et al (2005) Neuronal sorting protein-related receptor sorLA/LR11 regulates processing of the amyloid precursor protein. Proc Natl Acad Sci U S A 102:13461-13466

16. Dodson SE, Andersen OM, Karmali V, Fritz JJ, Cheng D, Peng J et al (2008) Loss of LR11/SORLA enhances early pathology in a mouse model of amyloidosis: evidence for a proximal role in Alzheimer's disease. J Neurosci 28:12877-12886

17. Rohe M, Carlo A-S, Breyhan H, Sporbert A, Militz D, Schmidt V et al (2008) Sortilin-related receptor with A-type repeats (SORLA) affects the amyloid precursor protein-dependent stimulation of ERK signaling and adult neurogenesis. J Biol Chem 283:14826-14834

18. Offe K, Dodson SE, Shoemaker JT, Fritz JJ, Gearing M, Levey Al et al (2006) The lipoprotein receptor LR11 regulates amyloid beta production and amyloid precursor protein traffic in endosomal compartments. J Neurosci 26:1596-1603

19. Rogaeva E, Meng Y, Lee JH, Gu Y, Kawarai T, Zou F et al (2007) The neuronal sortilin-related receptor SORL1 is genetically associated with Alzheimer disease. Nat Genet 39:168-177

20. Andersen OM, Rudolph I-M, Willnow TE (2016) Risk factor SORL 1: from genetic association to functional validation in Alzheimer's disease. Acta Neuropathol 132:653-665

21. Caglayan S, Takagi-Niidome S, Liao F, Carlo A-S, Schmidt V, Burgert T, et al (2014) Lysosomal sorting of amyloid- $\beta$ by the SORLA receptor is impaired by a familial Alzheimer's disease mutation. Sci Transl Med 6:223ra20

22. Nicolas G, Charbonnier C, Wallon D, Quenez O, Bellenguez C, GrenierBoley B et al (2016) SORL 1 rare variants: a major risk factor for familial early-onset Alzheimer's disease. Mol Psychiatry 21:831-836

23. Pottier C, Hannequin D, Coutant S, Rovelet-Lecrux A, Wallon D, Rousseau S et al (2012) High frequency of potentially pathogenic SORL1 mutations in autosomal dominant early-onset Alzheimer disease. Mol Psychiatry 17:875-879

24. Campion D, Charbonnier C, Nicolas G (2019) SORL1 genetic variants and Alzheimer disease risk: a literature review and meta-analysis of sequencing data. Acta Neuropathol 138:173-186

25. Schramm C, Charbonnier C, Zaréa A, Lacour M, Wallon D, CNRMAJ collaborators, et al (2021) Penetrance estimation of SORL 1 loss-of-function variants using a family-based strategy adjusted on APOE genotypes suggest a non-monogenic inheritance. bioRxiv. 2021.06.30.450554

26. Verheijen J, Van den Bossche T, van der Zee J, Engelborghs S, SanchezValle R, Lladó A et al (2016) A comprehensive study of the genetic impact 
of rare variants in SORL1 in European early-onset Alzheimer's disease. Acta Neuropathol 132:213-224

27. Holstege $H$, van der Lee $S J$, Hulsman M, Wong TH, van Rooij JG, Weiss M et al (2017) Characterization of pathogenic SORL1 genetic variants for association with Alzheimer's disease: a clinical interpretation strategy. Eur J Hum Genet 25:973-981

28. Vardarajan BN, Zhang Y, Lee JH, Cheng R, Bohm C, Ghani M et al (2015) Coding mutations in SORL1 and Alzheimer disease. Ann Neurol 77:215-227

29. Cuccaro ML, Carney RM, Zhang Y, Bohm C, Kunkle BW, Vardarajan BN et al (2016) SORL1 mutations in early- and late-onset Alzheimer disease. Neurol Genet 2:e116

30. Nicolas G, Wallon D, Charbonnier C, Quenez O, Rousseau S, Richard A-C et al (2016) Screening of dementia genes by whole-exome sequencing in early-onset Alzheimer disease: input and lessons. Eur J Hum Genet 24:710-716

31. Jack CR, Bennett DA, Blennow K, Carrillo MC, Dunn B, Haeberlein SB et al (2018) NIA-AA research framework: toward a biological definition of Alzheimer's disease. Alzheimers Dement 14:535-562

32. Karczewski KJ, Francioli LC, Tiao G, Cummings BB, Alföldi J, Wang Q et al (2020) The mutational constraint spectrum quantified from variation in 141,456 humans. Nature 581:434-443

33. Ran FA, Hsu PD, Wright J, Agarwala V, Scott DA, Zhang F (2013) Genome engineering using the CRISPR-Cas9 system. Nat Protoc 8:2281-2308

34. Concordet J-P, Haeussler M (2018) CRISPOR: intuitive guide selection for CRISPR/Cas9 genome editing experiments and screens. Nucleic Acids Res 46:W242-W245

35. Hayashi T, Lewis A, Hayashi E, Betenbaugh MJ, Su T-P (2011) Antigen retrieval to improve the immunocytochemistry detection of sigma-1 receptors and ER chaperones. Histochem Cell Biol 135:627-637

36. Schindelin J, Arganda-Carreras I, Frise E, Kaynig V, Longair M, Pietzsch T et al (2012) Fiji: an open-source platform for biological-image analysis. Nat Methods 9:676-682

37. Bolte S, Cordelières FP (2006) A guided tour into subcellular colocalization analysis in light microscopy. J Microsc 224:213-232

38. Kitago Y, Nagae M, Nakata Z, Yagi-Utsumi M, Takagi-Niidome S, Mihara E et al (2015) Structural basis for amyloidogenic peptide recognition by sorLA. Nat Struct Mol Biol 22:199-206

39. Rodrigues CH, Pires DE, Ascher DB (2018) DynaMut: predicting the impact of mutations on protein conformation, flexibility and stability. Nucleic Acids Res 46:W350-W355

40. Pettersen EF, Goddard TD, Huang CC, Couch GS, Greenblatt DM, Meng EC et al (2004) UCSF Chimera-a visualization system for exploratory research and analysis. J Comput Chem 25:1605-1612

41. Madsen P, Isaksen TJ, Siupka P, Tóth AE, Nyegaard M, Gustafsen C et al (2019) HSPA12A targets the cytoplasmic domain and affects the trafficking of the Amyloid Precursor Protein receptor SorLA. Sci Rep 9:611

42. Gustafsen C, Glerup S, Pallesen LT, Olsen D, Andersen OM, Nykjær A et al (2013) Sortilin and SorLA display distinct roles in processing and trafficking of amyloid precursor protein. J Neurosci 33:64-71

43. Shanthirabalan S, Chomilier J, Carpentier M (2018) Structural effects of point mutations in proteins. Proteins 86:853-867

44. Welsh MJ, Smith AE (1993) Molecular mechanisms of CFTR chloride channel dysfunction in cystic fibrosis. Cell 73:1251-1254

45. Schaeffer C, Creatore A, Rampoldi L (2014) Protein trafficking defects in inherited kidney diseases. Nephrol Dial Transplant 29 Suppl 4:iv33-44

46. Adams BM, Oster ME, Hebert DN (2019) Protein quality control in the endoplasmic reticulum. Protein J 38:317-329

\section{Publisher's Note}

Springer Nature remains neutral with regard to jurisdictional claims in published maps and institutional affiliations.

Ready to submit your research? Choose BMC and benefit from:

- fast, convenient online submission

- thorough peer review by experienced researchers in your field

- rapid publication on acceptance

- support for research data, including large and complex data types

- gold Open Access which fosters wider collaboration and increased citations

- maximum visibility for your research: over $100 \mathrm{M}$ website views per year

At BMC, research is always in progress.

Learn more biomedcentral.com/submissions 